\title{
Research On Dual-Carbon Services Based On Electric Vehicles And Pumped Energy Storage To Stabilize Power Fluctuations.
}

\author{
Weiyan Xu (D873124961@qq.com) \\ Yunnan Normal University \\ Zumin Liu \\ Yunnan Normal University \\ Jielei Tu \\ Yunnan Normal University
}

\section{Research Article}

Keywords: Carbon neutral, complementary landscape, Robustness, intelligence, environmental pollution

Posted Date: September 15th, 2021

DOl: https://doi.org/10.21203/rs.3.rs-850971/v1

License: (c) (i) This work is licensed under a Creative Commons Attribution 4.0 International License.

Read Full License 


\title{
Research on dual-carbon services based on electric vehicles and pumped energy storage to stabilize power fluctuations
}

\author{
Weiyan Xu * Zumin Liu Jielei Tu \\ *corresponding author(Weiyan $\mathrm{Xu}$ )
}

\author{
${ }^{1}$ Yunnan normal university \\ Author Xu Weiyan - Affiliation Yunnan normal university \\ Author Liu Zumin - Yunnan normal university \\ Tu Jielei - Yunnan normal university \\ Keywords: Carbon neutral, complementary landscape, \\ Robustness, intelligence, environmental pollution
}

\begin{abstract}
A B S T R A C T
Based on the research of electric vehicle energy storage stabilization, stochastic, adjustable, robust optimization, wind-solar complementary intelligent water pump system service double carbon, Which aims at: (1) In order to promote the consumption of wind power and photovoltaics in the grid, and reduce the pressure on the load of the distribution network; ( 2) Consider wind power and photovoltaic output fluctuations and electric vehicle cluster energy storage systems, and use multiple electric vehicle clusters to coordinate and smooth tie-line power fluctuations. The model includes: (1) a load monitoring terminal; (2) a server for storing, processing and mapping all collected electrical energy data; (3) a set of user-centric electrical energy management visualization and prediction services. (4) A set of photovoltaic water pumps, pumped energy storage systems and electric vehicles for supplying water to impoverished areas . Experimental data and practical applications show that because the wind and wind have a certain degree of complementarity, but are greatly affected by the climate, pumped energy storage and electric vehicles can reduce the volatility of the system. The research results show that photovoltaic and wind power have great volatility, and the current investment and operating costs of energy storage systems are relatively high. Largescale deployment of energy storage systems will seriously affect the economics of
\end{abstract}


photovoltaic and wind power into the grid. It is recommended that users can transfer part of the daily electricity load on Saturdays to Monday to Friday. The results also show that the wind and solar complementary system can improve the utilization of wind and solar energy, form a good complementarity, and the efficiency of the system is improved by $1.24 \%$. The addition of the wind-solar electric vehicle energy storage system has a certain impact on the operating cost of the system, and the grid-connected capacity is inversely proportional to the cost. When only thermal power is involved, the total operating cost of the system is 15.103 million yuan, and the total operating cost of the system after adding the wind-solar electric vehicle energy storage combined system is 876,000 yuan, and the total operating cost has dropped significantly. The power generation cost of wind turbines and pumped energy storage units is very small and can be ignored. The main cost of the system is the coal consumption cost of thermal power units and the start and stop costs of pumped energy storage units. After the combined wind storage system is added, the output of thermal power units in each period will be reduced. Small, the corresponding coal consumption is significantly reduced, so that the operating cost corresponding to each time period is significantly reduced.The overall situation is satisfactory. This paper studies the impact of thermal power units on carbon emissions, and the significance of energy transition and electric energy management to China's carbon neutrality . Every 1 kilowatt-hour of electricity replaced by a traditional thermal power unit is equivalent to saving 0.4 kilograms of coal energy and 4 liters of purified water. At the same time, it also reduces $1 \mathrm{~kg}$ of carbon dioxide and $0.03 \mathrm{~kg}$ of sulfur dioxide emissions. Liu Zuming's team built the world's first megawatt-level photovoltaic water pumping system. Take this as an example to calculate. Based on $5000 \mathrm{kWh}$ a day, it can reduce 5 tons of carbon dioxide emissions per day. According to photovoltaics, they can be used for 20 years, at least. Can reduce 36500 tons of carbon dioxide emissions. In addition, his team has built more than 160 photovoltaic power plants in the Yunnan Plateau, which can have a longer-term and comprehensive impact on carbon emissions. Jiangyi Township's emergency water supply for drought relief and rural drinking water safety consolidation and improvement of the project's annual water supply The volume is $410,800 \mathrm{~m} 3$, covering 
6 village committees and 41 natural villages in Jiangyi Township, Jiangyi, Painter, Banqing, Gongcha, Horizontal Stone, and Nuolakun, and solves the problem of safe drinking water for 11,590 people and 3140 large livestock in Jiangyi Township. problem. In conclusion, the experimental evidence introduced in this article supports that the random adjustable robust optimization of wind-solar complementary smart pump home energy management system is feasible and valuable for residents in poverty-stricken areas and China, and the impact of energy transition on China's carbon neutrality is significant and far-reaching.

\section{Introduction}

As we all know, energy transformation is one of the most powerful measures to solve carbon emissions, Because a large amount of carbon dioxide emissions can lead to the greenhouse effect. Despite the increasing awareness of citizens and governments,Policymakers also recognize the importance of reducing carbon dioxide emissions, At present, China's greenhouse gas emissions are about 14 billion tons, and energy related carbon dioxide emissions account for $73 \%$ of all greenhouse gas emissions. It still fails to meet the requirements of China's carbon peak before 2030 and carbon neutralization before 2060(outline of China's 14th Five -year plan). Therefore, It is very important for us to actively transform energy's type to strengthen carbon emission reduction. To devise such strategies, We need to improve the effectiveness of the energy management system to reduce energy consumption and improve energy efficiency continuously. We will continue to reduce energy consumption and carbon dioxide emissions by building a green energy management system, promoting renewable energy to replace traditional energy, and developing technology and energy conservation management. that is, We will conduct on-the-spot investigation on the current energy structure and energy management mode. In 2020, the number of electric vehicles in my country will reach $5 \times 10^{\wedge} 6$, and large-scale electric vehicles will be connected to the network, and the impact capacity will be very considerable. Fully consider the travel laws of electric vehicles and evaluate their available capacity. At the 
same time, optimize the wind and solar coordinated dispatch target.

Carbon dioxide and other greenhouse gases have attracted increasing attention, Because these have a significant impact on human environment. Therefore, the trend of global warming must be curbed. Energy must be transformed, However, although new energy sources have less pollution to the environment, But wind power and photovoltaic power generation are affected by weather and other factors greatly. Mainly because of the volatility and randomness of wind and solar energy. As a result, Single wind energy and solar energy systems are less stable and have a greater impact on the power grid. In addition, the randomness of photovoltaic and wind power generation has caused more serious wind and solar abandonment. The result is a waste of energy. For this reason, it is possible for electric vehicles and pumped energy storage to participate in grid dispatching. By optimizing the charging and discharging process of electric vehicles, it is possible to promote the consumption of distributed power sources, Compared to traditional single energy research and application, Wind solar complementary system and effective energy management have a great of significance to accelerate carbon neutralization, improve energy utilization and increase the income of poor areas. The new energy management optimization technology has had a profound and lasting impact on reducing carbon dioxide emissions. For example, intelligent prediction algorithm provides a feasible solution for developing energy management system. Robust optimization is the objective function. Under certain constraints, no matter how other uncertain parameters change in the solution set, it can ensure the reliability and stability of the system and realize the optimization of the objective function in the most extreme cases. According to the Copula theory, the Frank Copula function of the negative correlation structure is selected as the connection function of the joint probability distribution of wind farms and photovoltaic power plants. In practical applications, the forecasts of wind power and photovoltaics can be updated at any time in the actual situation. Nevertheless, in order to obtain high-quality prediction data, a high-precision prediction algorithm is required. According to the Copula theory, the reliability and stability of the system are guaranteed (Sklar, 1959) . 
As part of the National Natural Science Foundation of China, the main contribution of the current research is to solve the problem of drinking water and energy waste in impoverished areas and improve the utilization of idle land resources in impoverished areas. Improved and optimized the robustness of wind power and photovoltaics. Increased the economic output of crops in impoverished areas. Reduce the large amount of carbon dioxide emissions caused by thermal power units. By collecting the local weather forecast data, combined with the historical power generation and power load data, the prediction optimization is carried out. Ensure that the energy utilization of the system is maximized and the robustness of the system is guaranteed. The core of the research is to enhance the safety and reliability of the power grid and improve the utilization of wind and light energy, However, different from most studies now, our research is mainly aimed at poor and remote areas. Our system needs to be able to supply power to local residents in extreme weather conditions. Wind power grid integration increases the spinning reserve capacity to compensate for the uncertainty of wind power output, so this paper introduces spinning reserve penalty costs. The extreme penetration of wind power often causes abandonment of wind farms, resulting in waste of resources and low wind power utilization. Therefore, this paper introduces the cost of abandonment penalty.

Although the idea of wind-solar hybridization was developed by Xinjiang TBEA New Energy Company to build my country's first large-scale wind-solar hybrid power station of 100 megawatts (2013) , But these early solutions did not involve energy management. Our research also included electric vehicles and photovoltaic water pumps. Its mission is to collect historical weather, electricity energy data, People's Daily habits and typical load, to provide people with a set of energy efficiency maximization, revenue maximization, stable and reliable quality electricity service.Our research aims to improve local residents' fossil energy easily depleted and the resulting air pollution and greenhouse effect damage our living environment. Therefore, the research on the energy management system of the household energy management system with random adjustable robust optimization wind-solar complementary 
intelligent water pump helps people in poverty-stricken areas to change their behaviors and arrange daily electricity consumption according to the prompts of the electric energy data management system, which not only greatly improves our lives The environment, income from drinking water, agriculture, and animal husbandry for local residents have also been significantly improved and increased. In addition, the system also allows residents to determine the online time for the generated energy based on the peak, flat, and valley electricity prices, so as to maximize the benefits for local residents. How to improve the ecological environment, quality of life, drinking water in povertystricken areas, and increase energy efficiency are the main concerns of this research.

In summary, the system we studied provides a novel and practical solution for energy management and drinking water in poor areas, reducing the emissions of carbon dioxide and toxic gases produced by thermal power plants and fossil fuels. Together with a set of practical photovoltaic water pumping system, it can solve the water problem of people, livestock and crops in poor areas. The chapter structure of this article is arranged as follows. In the second section, the stochastic adjustable robust optimization wind-solar complementary intelligent water pump family energy management system is described. Section 3 presents the results and verifies the system in a set of field experiments. Finally, the fourth part summarizes the conclusions and proposes future work directions.

\section{Proposed system}

\subsection{System architecture}

The energy management system proposed in this paper is a hierarchical, distributed intelligent system, whose purpose is to make a power generation plan, electricity use plan, water use plan, and energy storage plan in advance based on local weather forecasts to optimize energy utilization. To provide citizens in poverty-stricken areas with intuitive electricity consumption planning, integrate these data together, and cultivate a more scientific way of electricity consumption. In order to achieve these goals, the system is divided into three layers, as shown in Figure 1: 


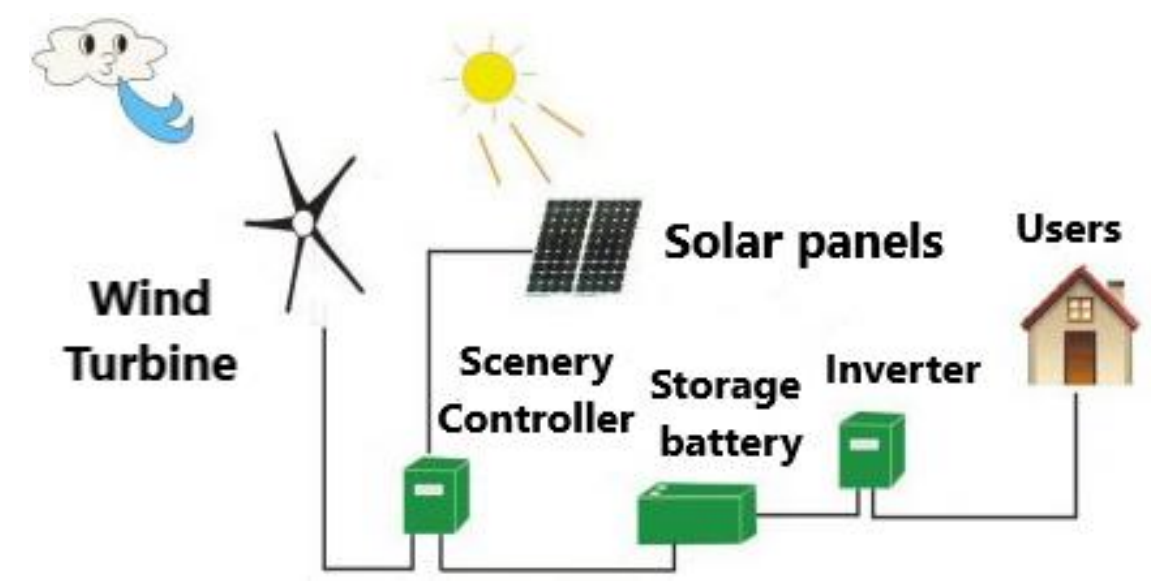

Fig. 1. Wind, solar and water complementary system

First of all, due to the intermittence and fluctuation of photovoltaic and the great impact of climate, it is difficult to ensure stable power output, while solar energy and wind energy have natural complementarity. With the help of wind solar complementary power generation system, it can effectively increase the penetration rate of new energy, improve the controllability of output power and improve the reliability of grid connected power generation of new energy.

The energy management system is responsible for collecting all electric energy data and local weather data, predicting and using them, make a reasonable power consumption plan for local residents. The wind solar complementary robust optimal scheduling model based on Copula theory is adopted to ensure the robustness of the system. The problem of water for people, livestock and crops in poor areas has been solved. Make an accurate prediction and reasonable power consumption and generation plan by using historical power consumption and generation data, weather forecast, water consumption and electric vehicle travel. The energy storage system can control the active power output by the wind power generation. The energy storage system can not only be used for power peak shaving, smooth the power output of the wind farm, and make the wind unit operate as a dispatch unit unit, but also has the ability to provide frequency control to the power system. Auxiliary service functions such as fast power response, taking full consideration of the mobile energy storage characteristics of electric vehicles, using electric vehicle internal battery supercapacitors, combined with pumped energy storage technology, can not only provide high-quality electrical energy 
to the grid, but also increase wind power and photovoltaics The operating efficiency of the wind power and photovoltaic power in the energy market will be improved.

Photovoltaic power generation and wind power generation have natural complementarity and show weak nonlinear correlation. It is very difficult to determine the relationship between them by using the traditional probability theory. The introduction of Copula theory provides a new method to solve this problem.In practice, the output of wind farms and photovoltaic power stations in the same region are complementary. Therefore, the characteristic Frank copula function of negative correlation structure is selected as the connection function of the joint probability distribution of wind farm and photovoltaic power station. The distribution function and density function of Frank copula function are:

$$
\begin{aligned}
& C(u, v ; \theta)=-\frac{1}{\theta} \ln \left[1+\frac{\left(\mathrm{e}^{-\theta u}-1\right)\left(\mathrm{e}^{-\theta v}-1\right)}{\mathrm{e}^{-\theta}-1}\right](1) \\
& c(u, v ; \theta)=\frac{-\theta\left(\mathrm{e}^{-\theta}-1\right) \mathrm{e}^{-\theta(v+u)}}{\left[\left(\mathrm{e}^{-\theta}-1\right)+\left(\mathrm{e}^{-\theta u}-1\right)\left(\mathrm{e}^{-\theta v}-1\right)\right]^{2}}
\end{aligned}
$$

In the formula, $u 、 v$ the output of wind farm and photovoltaic power station is respectively represented, $\theta$ which is a relevant parameter. At that time $\theta \neq 0, \theta>0$, $u$ 、 $v$ it represents the output and shows a positive correlation; At that time $\theta \rightarrow 0, u$ 、 $v$ it means output and tends to be independent; At that time $\theta<0, u 、 v$ it means output and negative correlation.

\subsubsection{Economic Cost of Wind Farm}

As a representative of new energy, wind power has the characteristics of no pollution and no coal consumption, but the intermittent nature of wind farms will seriously affect the safety and reliability of the power grid. The grid-connected wind power increases the spinning reserve capacity to make up for the uncertainty of wind power output[8], Therefore, this article introduces the penalty cost of spinning reserve 。 The extreme penetration of wind power often causes abandonment of wind farms, resulting in waste of resources and low wind power utilization. Therefore, this paper introduces the cost of abandonment penalty. Most of the costs of wind farms are in the 
construction phase, so relatively small operating costs are ignored here. (3) The penalty cost of wind farm spinning reserve capacity is:

$$
C_{\text {pu. } t}=\sum_{j=1}^{n} C_{\text {re. } t}\left(1-E_{\text {cre. } . t}\right) P_{\text {re. } j t}
$$

Where $j$ Respectively $n$ Number of wind farms; $C_{\text {pu.t } t}$ Indicates the cost of spinning reserve capacity per unit time of wind farms; $\mathrm{C}_{\text {re.t }}$ For wind farms in $t$ The price of spare capacity during the time period, $\mathrm{E}_{\text {cre. } j t}$ Wind farm $j$ exist Reliability over time, $P_{\text {re.jt }}$ Wind farm $j$ exist Forecast output over time。

(2) The penalty cost of wind farm abandonment is:

$$
C_{\text {lpp.t }}=\left\{\begin{array}{l}
\sum_{j=1}^{n} C_{m . t}\left(P_{\text {plan.jt }}-P_{\text {re. } j t}\right) ; P_{\text {re. } j t}<P_{\text {lpp.jt }} \\
\sum_{j=1}^{n} C_{m . t}\left(P_{\text {plan.jt }}-P_{\text {lpp. } j t}\right) ; P_{\text {re. } j t} \geq P_{\text {lpp. } j t}
\end{array}\right.
$$

Where $j$ Respectively $n$ Number of wind farms, $C_{m . t}$ Wind farm $j$ exist $t$ Unit penalty cost during the time period, $P_{\text {plan.jt }}$ Wind farm $j$ exist $t$ Planning and dispatching output within the time period, $P_{l p p . j t}$ Wind farm $j$ exist $t$ Limit penetration power in time period, $C_{l \mathrm{pp} . t}$ Indicates the penalty cost of abandoning wind per unit time of the wind farm。

(3) Economic cost of wind farm:

$$
C_{\mathrm{w} . t}=C_{\mathrm{pu} . t}+C_{\mathrm{lpp} . t}
$$

Where $\mathrm{C}_{\mathrm{w} . t}$ Wind farm $t$ The total cost during the time period.

\subsubsection{Economic cost of photovoltaic power station}

Photovoltaic power generation is greatly affected by the weather and environment, Large scale photovoltaic grid connection requires thermal power units to increase rotating reserve capacity to compensate for power fluctuation caused by output change, In this paper, the intermittent change of light intensity is transformed into the penalty cost of photovoltaic power generation。Because the operation and maintenance cost is relatively low and most of the costs are in the construction stage, this paper only considers the penalty cost of rotating reserve capacity。 The economic cost of photovoltaic power station .

Where $k$ Indicates the number of photovoltaic power station, $P_{v k a}$ Represents the planned power generation of the photovoltaic power plant, $C_{\mathrm{pv}}\left(P_{\mathrm{via}}\right)$ Represents the 
economic output cost of photovoltaic power station in unit time, $N_{r p}$ Represents the penalty cost coefficient of rotating reserve capacity of photovoltaic power station, $C_{m} \mathrm{It}$ represents the economic output cost function of thermal power unit with the highest unit output cost at the maximum efficiency, $P_{v k}$ Represents the actual output power of the photovoltaic power station.

On the basis of considering environmental pollution and fuel loss, combined with the formula (5) $\sim$ (11) The objective function of minimizing the total power generation cost per unit cycle is proposed. $\min C=\sum_{t=0}^{T}\left[\sum_{i=1}^{I} C_{i}\left(P_{i}\right)+\sum_{k=1}^{K} C_{\mathrm{pv}}\left(P_{v k a}\right)+C_{\mathrm{w} . t}\right]$

Where $T$ Indicates the number of cycles per unit, $I 、 K$ Respectively indicate the number of thermal power plants and photovoltaic power plants in the system within a period. The family energy management system is shown in Figure 2.

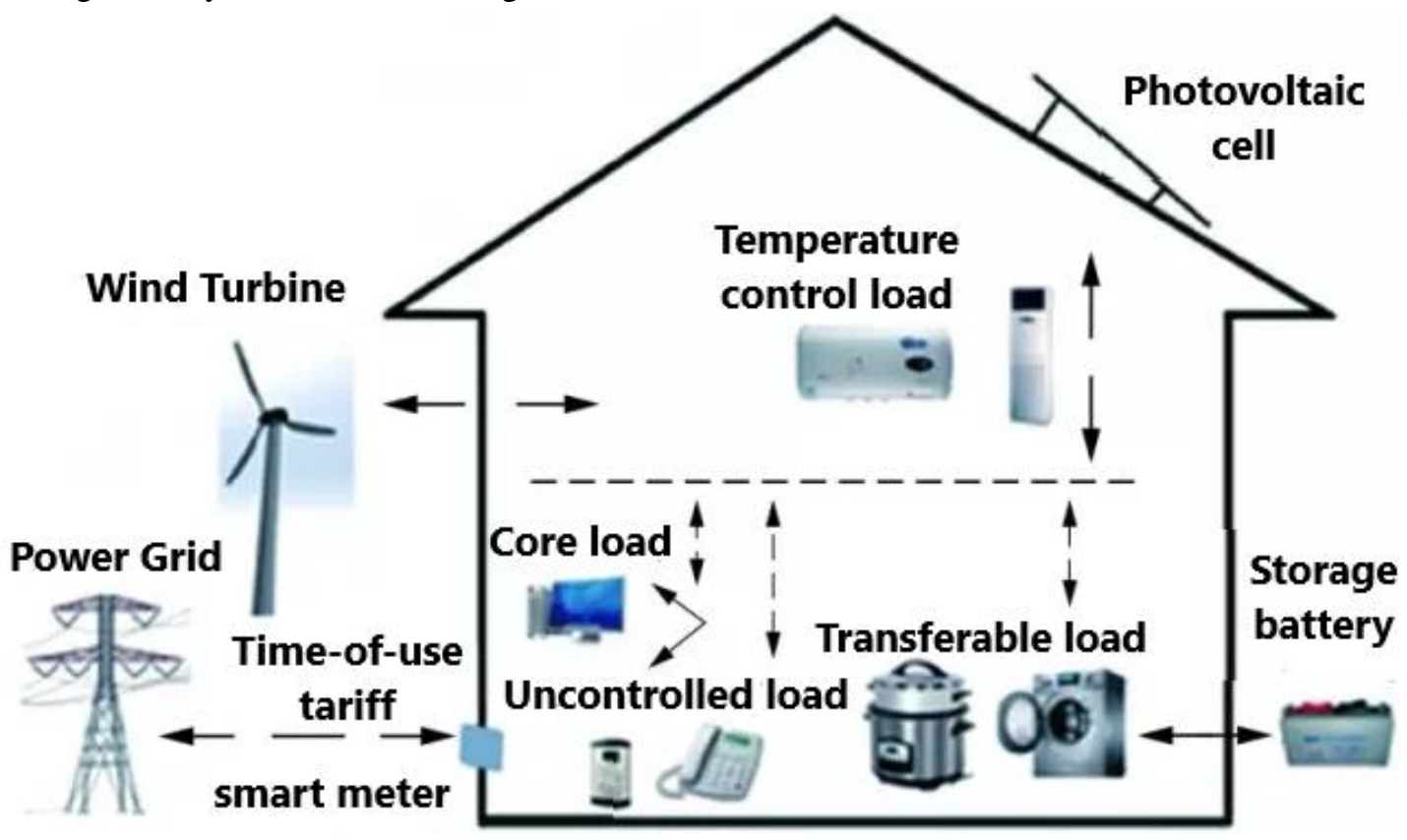

Fig.2. Schematic diagram of energy management system structure

The output combination of wind farm and photovoltaic power station with windsolar complementary independent operation configuration takes advantage of the natural characteristics of wind-solar complementary to a certain extent, and the fluctuations of the power into the network will be improved when the grid is connected. The reliability and security of the grid should be ensured, and the fluctuations of the power into the network should be small [9]. In this paper, the wind-solar complementarity characteristics are fully considered, and the reliability optimization strategy of wind-solar complementarity is adopted to configure the output power and rotary reserve capacity of photovoltaic power stations and wind farms under the condition that the economic cost of the objective function is small. 
Wind power generation and photovoltaic power generation are greatly affected by the environment. In order to measure the correlation degree of wind-solar complementarity, the correlation change rate of wind-solar complementarity is defined in this paper $\lambda$ 。

$$
\lambda=\frac{\mathrm{d} \tau}{\mathrm{d} t}
$$

In this paper, the credibility of wind-solar complementarity is defined by fuzzy opportunity constraint, and the credibility measure of wind-solar complementarity can be solved by possibility measure. For the possibility space, the credibility of the event is expressed as [10-12]:

$$
\operatorname{Cr}\{A\}=\frac{1}{2}\left(\operatorname{Pos}\{A\}+1-\operatorname{Pos}\left\{A^{-1}\right\}\right)
$$

Where $C r\{A\}$ Show event $A$ Credibility, $\operatorname{Pos}\{A\}$ Show event $A$ Show event, $A^{-1}$ Show event $A$ Opposing events 。

In order to define the power fluctuation of the wind-solar hybrid grid-connected system, this paper introduces the credibility into the wind-solar hybrid system and defines the confidence level $\delta$, Expressed as:

$$
\delta \leq \operatorname{Cr}\{|\lambda| \leq \varepsilon\}
$$

Where $\varepsilon$ Indicates the minimum acceptable change rate of wind output 。

According to formula (22), Figure 1 can be obtained, It can be seen from Figure 1: With the Kendall rank correlation coefficient $\tau$ With the increase of the negative correlation of the wind and solar hybrid system, the confidence level of the wind and solar hybrid system continues to increase; as the number of power stations $\mathrm{M}$ increases, the confidence level of the wind and solar hybrid system also shows a weak increase.

Fig.1 Relationship between $\tau, M$ and $\delta$ of Kendall rank correlation coefficient,According to the definition of the robust optimal scheduling model, the expression of the wind-solar complementary robust optimal scheduling model in this paper. It can be seen that the robust optimal scheduling model in this paper is a classic nonlinear quadratic programming problem, and this paper uses optimization dual internal point generation to solve it.

\subsubsection{Analysis of calculation examples}

In order to verify the application of the system in actual scenarios, a simplified power system of 2 wind farms and 2 photovoltaic power plants was used as an example to conduct optimization dispatch analysis and confidence level analysis of wind-solar hybrid power generation system. Use 80MW photovoltaic power plant and $80 \mathrm{MW}$ wind farm. Considering the actual local situation, this paper chooses the study period from 8:00 to 18:00, and the power 
output forecast is shown in Table 1. Considering the actual local situation, this paper chooses the study period from 8:00 to 18:00, and the power output forecast is shown in Table 1. The robust optimization theory is used to deal with the uncertain variables in the wind-solar hybrid system, which enhances the robustness of the system.
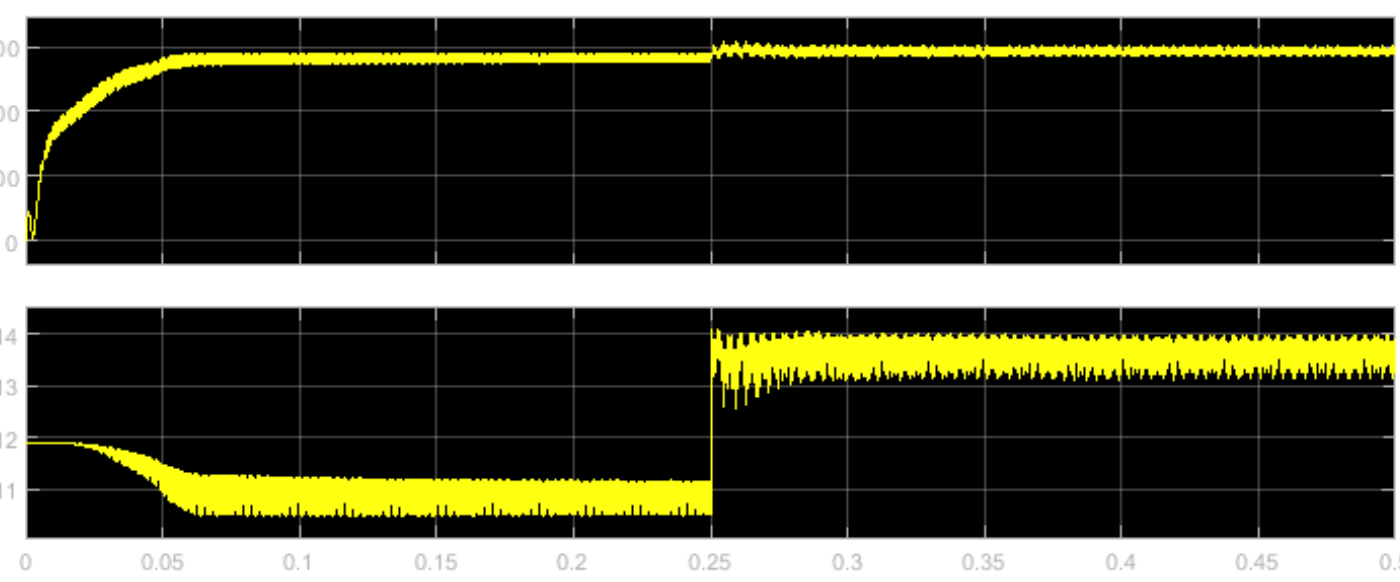

Fig.3.Curve diagram of PV forecast output

Fig.3. shows the relationship between the number of power stations and confidence level in the wind solar complementary system. It can be seen that with the increase of the number of power stations, the confidence level increases accordingly, indicating that the number of power stations plays a supporting role in the safety and reliability of the whole power grid and plays a vital role in the robustness of the whole power grid. Figure 6 shows the output comparison diagram of wind solar complementary system under different confidence levels. It can be seen that with the improvement of confidence level, the power output of wind solar complementary system is more stable. This is because the greater the negative correlation of wind solar complementary system, the more significant the output power of wind power generation and photovoltaic power generation has the characteristics of mutual compensation. Robust optimal scheduling also takes into account the randomness and intermittence of photovoltaic output and wind farm output, making the scheduling results more robust. The effect diagram of different confidence intervals is shown in Figure 4. 


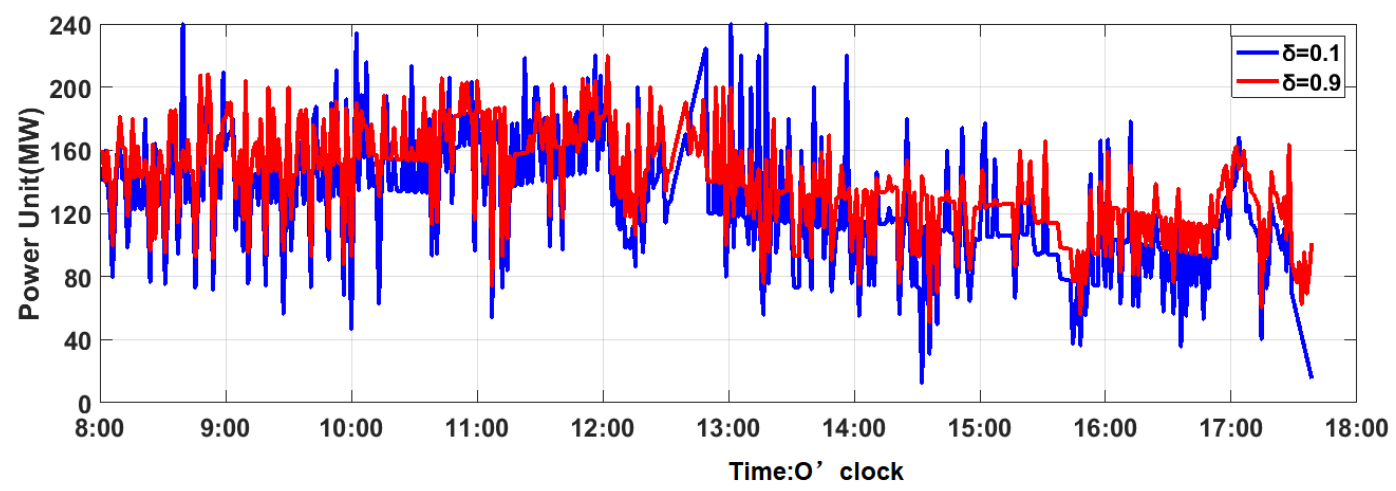

Fig.4.Relationship between $M$ and $\delta$, The results show that the proposed algorithm is robust when $\delta=0.9$

Because when the change rate of the total output of the photovoltaic power plant and the wind farm fluctuates greatly, the robust optimal scheduling introduces the confidence level into the decision strategy under the condition of considering the correlation, intermittence and uncertainty of the wind farm and the photovoltaic power plant, which makes the decision strategy more robust. When the confidence level is 0.1 , it shows that the scheduling strategy does not consider the uncertainty of the output of each electric field, the output of each photovoltaic power station and wind farm is the expected output, the output of each power station is regarded as a fixed value, and no scheduling strategy is made. The robust optimal scheduling under the power grid confidence level is the traditional economic scheduling without considering the output uncertainty. With the improvement of confidence level, the consideration of 
uncertainty of photovoltaic power plant and wind farm will gradually increase. When the confidence level is 0.9 , the scheduling strategy will fully consider the intermittency and complementarity of

2.3 Intelligent optimal dispatching of electricity load in rural households

Through load-side management, household energy efficiency is improved and electricity costs are saved. ] Establish an equivalent model for the temperature control load in the family, and dispatch it with the goal of demand response optimal dispatch. In view of the characteristics of new energy output forecast accuracy, random optimization and robust optimization are combined to give play to the advantages of both to promote the consumption of new energy in the grid. At present, there are few literatures on the application of this hybrid method in the optimal scheduling strategy of household smart electricity consumption while considering the uncertainty of users' electricity consumption behavior.

Next, we combine the optimal dispatch of rural household electricity load to study. According to the operating characteristics of the household electricity load and its controllability for the household energy management system, the article divides the household electricity load into the following four categories: Basic load, Unschedulable flexible load, schedulable uninterruptible load, temperature control load. The household energy management system needs to consider the uncertainty of users' electricity consumption behavior in the decision-making process. The model is shown in formula (9):

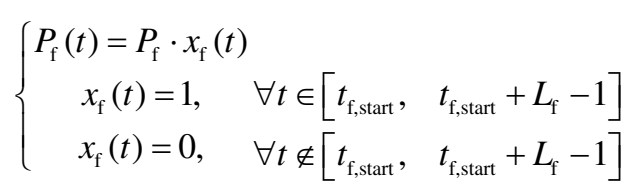

Where $P_{\mathrm{f}}(t)$ for $t$ Unschedulable flexible load power consumption in a time period; $P_{\mathrm{f}}$ The power consumed when the load is running; $x_{\mathrm{f}}(t)$ for $t$ The operating status of the load during the time period; $t_{\mathrm{f}, \text { start }}$ and $L_{\mathrm{f}}$ It is the initial running time and rated running time of a task for this load. 
Washing machines, dryers, dishwashers, etc. are schedulable and uninterruptible loads with fixed working cycles and a certain degree of flexibility in running time. They can be scheduled by the home energy management system. After startup, they must run continuously until the task is completed. The model is not schedulable. The form of the flexible load model is the same. Scheduling this type of load will not significantly restrict users' daily life behaviors.

Generally speaking, as the distance from the operating point increases, the prediction accuracy of photovoltaic power generation output gradually decreases. The short-term forecast of photovoltaic power output $(0 \sim 72 \mathrm{~h})$ has an error of $5 \% \sim 25 \%$, mostly concentrated in $10 \% \sim 20 \%$, while the error of ultra-short-term forecast $(0 \sim 4 \mathrm{~h})$ can be less than $1 \%[19-21]$. Unlike wind power, the photovoltaic power generation system only generates electricity during the day and the output power at night is 0 . Therefore, when HEMS formulated the scheduling strategy for the second day the night before, the forecast of the output of the distributed home grid-connected photovoltaic system on the second day was obtained. The value has a large uncertainty, and the photovoltaic power output of the time period closer to the operating point (such as the early morning period of the next day) is a certain value of 0 . In order to ensure the flexibility and freedom and comfort of users, promote the local consumption of photovoltaic and wind power while minimizing the operating cost of the system. In the time period close to the time point of formulating the scheduling strategy, the output of photovoltaic power generation is a certain value of 0 , and the prediction accuracy of the user's electricity consumption behavior is relatively high. The PSO training diagram is shown in Figure 5 and Figure 6. 


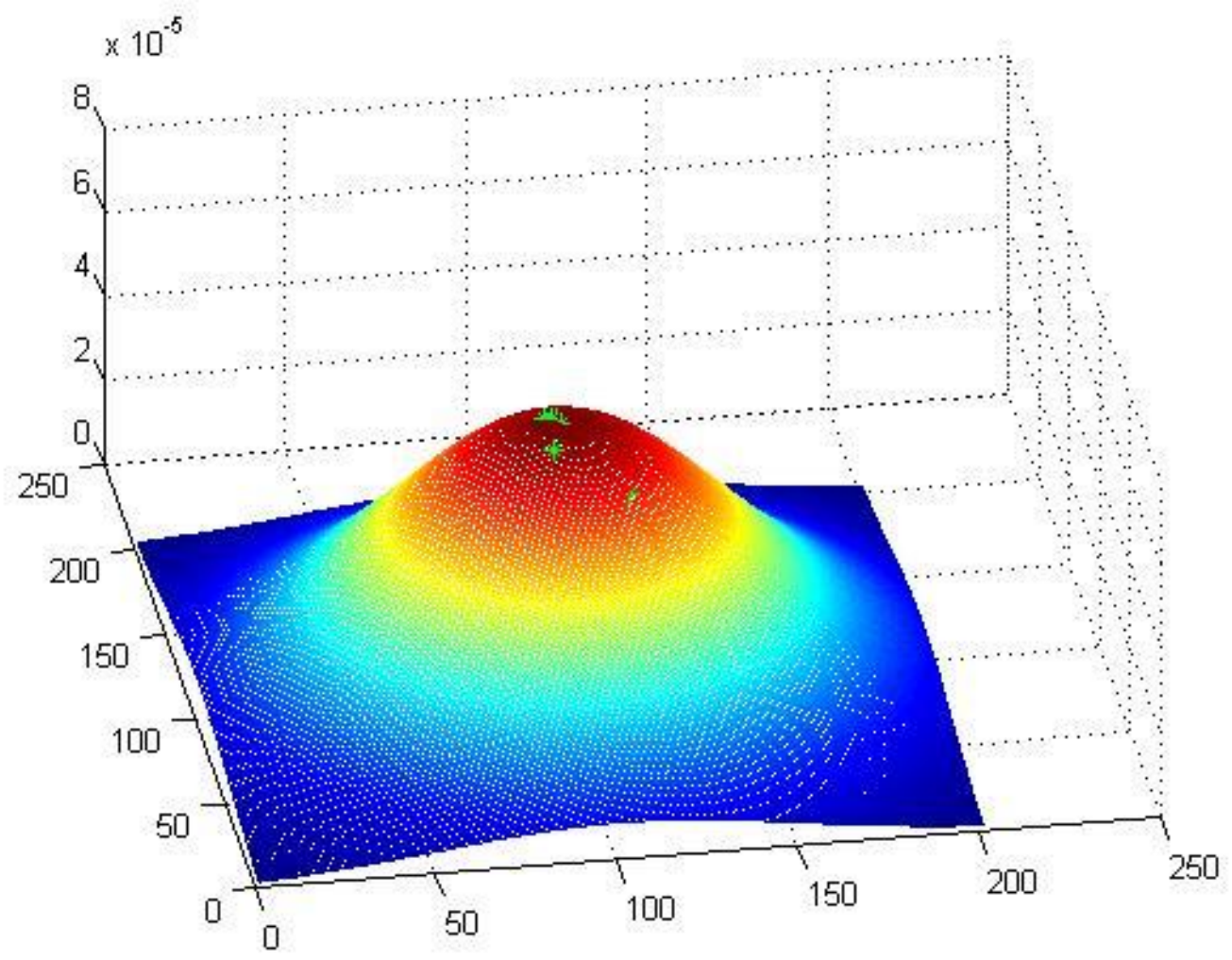

Fig.5. PSO training Gaussian function graph, the green dot is the optimal position

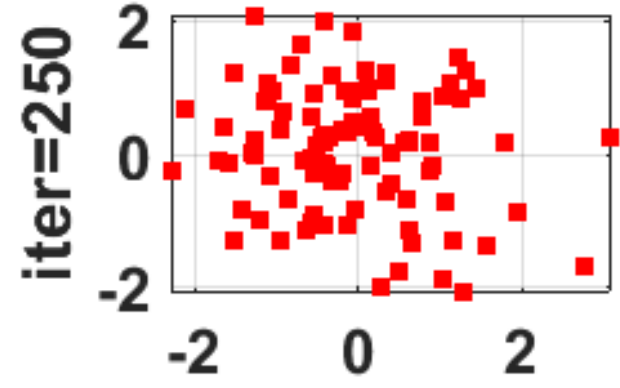

(a)

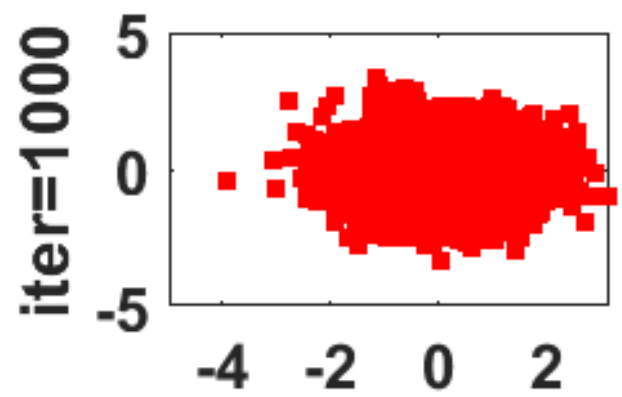

(c)

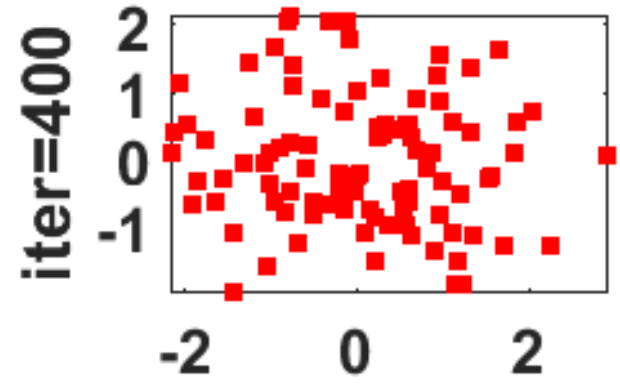

(b)

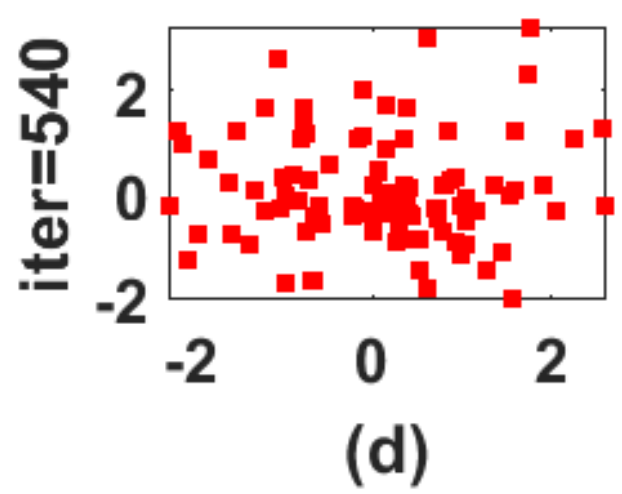

Fig.6. Training scatter plots of different algebras 
The system scheduling period in the article is $\mathrm{T}=24 \mathrm{~h}$, which is equally divided into $\mathrm{N}=96$ time periods, and each time period is $\Delta \mathrm{t}=15 \mathrm{~min}$. In the example system, the distributed power supply includes a distributed photovoltaic power generation system with a total capacity of $2.5 \mathrm{~kW}$ and an energy storage system with a total capacity of $10 \mathrm{~kW} \cdot \mathrm{h}$. The remaining parameters of the energy storage system are shown in Table 1. Tab.1 Parameters of energy storage system

\begin{tabular}{cccc}
\hline$S O C_{\min }$ & $S O C_{\max }$ & $\eta_{\mathrm{ch}}$ & $\eta_{\text {dis }}$ \\
\hline 0.1 & 0.9 & 0.95 & 0.95 \\
\hline$E_{\mathrm{B}}(0) /(\mathrm{kW} \cdot \mathrm{h})$ & $P_{\mathrm{ch}, \max } /(\mathrm{kW})$ & $P_{\mathrm{dis}, \max } /(\mathrm{kW})$ & $c_{\mathrm{op}} /($ 元 $/ \mathrm{kW} \cdot \mathrm{h})$ \\
\hline 5 & 2.5 & 2.5 & 0.15 \\
\hline
\end{tabular}

The predicted expected value and fluctuation range of photovoltaic power generation output on the next day and outdoor temperature are shown in Figure 1 and Figure 2 respectively. The sunrise time of the next day is 06:00 and the sunset time is 19:30, so the scheduling strategy uses $06: 00$ as the switching point between random optimization and adjustable robust optimization. Among the parameters of the air conditioning system, $\mathrm{R}, \mathrm{C}, \mathrm{Q}$, Tset, and $\sigma$ are respectively $12.8{ }^{\circ} \mathrm{C} / \mathrm{kW}, 0.36 \mathrm{~kW} \cdot \mathrm{h} /{ }^{\circ} \mathrm{C}$, $1.5 \mathrm{~kW}, 26{ }^{\circ} \mathrm{C}$, and $2{ }^{\circ} \mathrm{C}$, and the initial indoor temperature is $26{ }^{\circ} \mathrm{C}$. In the calculation example, the price of electricity sold by the user to the grid is $0.34 \mathrm{yuan} / \mathrm{kW} \cdot \mathrm{h}$, and the price of electricity purchased from the grid is the peak-to-valley price, and the peakhour $(10: 00-15: 00,18: 00-22: 00)$ price is $0.9 \mathrm{Yuan} /(\mathrm{kW} \cdot \mathrm{h})$, the electricity price during valley hours $(00: 00-07: 00)$ is $0.3 \mathrm{yuan} /(\mathrm{kW} \cdot \mathrm{h})$, the rest of the time is normal, the electricity price is $0.5 \mathrm{yuan} /(\mathrm{kW} \cdot \mathrm{h})$.

The random optimization stage is from 00:00-06:00 the next day. In this stage, the photovoltaic output is 0 , the load is lighting fixtures and hot water boilers, and the power is $0.25 \mathrm{~kW}$ and $1.8 \mathrm{~kW}$, respectively. Cut it into three scenes, the probabilities are $0.3,0.5,0.2$. The decision variables at this stage are the charge/discharge power of the energy storage system, the on-off state of the air-conditioning system, and the power supply state of the fan. The constraint range of $\mathrm{EB}(\mathrm{tsw})$ at the end of random optimization is further reduced, and $\mathrm{k}$ is 0.1 in formula (26).

In this paper, the CPLEX solver is called to solve the MILP problem through the YALMIP toolbox in MATLAB. 
If an adjustable robust optimization method is used to formulate a scheduling strategy for the entire scheduling cycle (ie $0-24 \mathrm{~h}$ ), the total operating cost of the system is 3.481 yuan, of which the total cost for the 00:00-06:00 time period is 0.1578 yuan, and the electricity purchase The cost is 0.6337 yuan, the battery life loss cost is 0.426 1 yuan, and the electricity sales income is 0.902 yuan. Compared with the random \& adjustable robust optimization hybrid scheduling proposed in the article, the one-stage optimization method that uses pure robust optimization for the entire scheduling cycle takes into account the use of users with a small probability of occurrence during the 00:00-06:00 time period. In extreme cases of electrical behavior, the random \& adjustable robust optimization proposed in the article is aimed at the characteristics of less uncertainty in the 00:00-06:00 time period, and adopts a scenario-based random optimization method, which effectively reduces the operating cost of the system. Improve the economic efficiency of household electricity.

\subsubsection{Pumped energy storage unit model and constraints}

The power generation of a pumped energy storage power station in operation is not only related to the number of generating units and each output, but also needs to be less than the reservoir energy storage (in $\mathrm{MW} \cdot \mathrm{h}$ ) that the power station can provide, as shown in equation (11). The pumping mode unit runs at rated power $\mathrm{PpN}$, and the total pumping power needs to be less than the wind power during this period and the energy storage corresponding to the remaining water storage space of the pumped energy storage power station, as shown in equation (12). The unit cannot be in the state of pumping water and generating electricity at the same time, and the design constraints are shown in equation (13). In addition, the total number of pumped generator sets $\mathrm{N}$ remains unchanged, and the number of start and stop units can be calculated according to the number of units N0t in shutdown conditions at each time period, as shown in equations (14), (15) and (16).

$$
\begin{aligned}
& P_{\min }^{g} k_{t} \leq P_{t}^{g} \leq \min \left(P_{\text {max }}^{g} k_{t}, \frac{\left(E_{t-} E_{\text {min }}\right) \eta_{g}}{\nabla t}\right) \\
& P_{t}^{p}=P_{N}^{P} U_{t} \leq \min \left(P_{t}^{w}, \frac{E_{\max }-E_{t}}{\Delta t \eta_{p}}\right) \\
& P_{t}^{p} P_{t}^{g}=0 \\
& N=k_{t}+u_{t}+N_{t}^{0} \\
& n_{t}^{s u}=\max \left(N_{t-1}^{0}-N_{t}^{0}, 0\right)
\end{aligned}
$$




$$
n_{t}^{s d}=\max \left(N_{t}^{0}-N_{t-1}^{0}, 0\right)
$$

In the formula, $P{ }_{t}^{p}$ and $P_{t}^{g}$ are the power generation and pumping power of the pumped-storage unit in period $\mathrm{t}$, respectively; $P_{t}^{w}$ is the power generated by the wind turbine in period $\mathrm{t}, P_{\min }^{g}$ And $P_{\max }^{g}$ are the minimum and maximum power generation of a single unit, respectively, in MW; $k_{t}$ and $u_{t}$ are the number of units in power generation and pumping conditions during the $\mathrm{t}$ period, respectively; $E_{t}$ is the energy storage of the upper reservoir at the beginning of the t period, $\mathrm{MW} \cdot \mathrm{h} ; \eta \mathrm{p}$ and $\eta g$ are the pumping and power generation efficiency of the unit respectively; $n_{t}^{s u} n_{t}^{s d}$, Respectively the number of units starting and stopping during t period; $E_{\min }$ and $E_{\text {max }}$ are the minimum and maximum energy storage available in the pumped storage power station reservoir, MW'h. With the goal of minimizing the cost of the combined operation of wind power and pumped energy storage, considering the start-up and shutdown costs of the generator set in the pumped energy storage power station, the objective function is:

$\min \sum_{i=1}^{T}\left[C_{s u} n_{t}^{s u}+C_{s d} n_{t}^{s d}+\sum_{i=1}^{N_{t}} f(i)\right]$

In the formula, $C_{s u}$ and $C_{s d}$ are the number of units starting and stopping; $\mathrm{f}(\mathrm{i})$ is the fuel cost of a conventional unit. The constraints are as follows:

System power balance constraints.

$\sum_{k=1}^{N_{W}} P_{W k t}+\sum_{j=1}^{N t} P_{H j t}+\sum_{i=1}^{N_{i}} u_{i t} P_{G i t}=P_{D t} \quad$ (18)

In the formula, $N_{w}$ is the total number of wind farms; $P_{W k t}$ is the total output of all units in the wind farm k during period t; $P_{D t}$ is the load value during period t; $N_{t}$ is the number of pumped energy storage units; $P_{H j t}$ is the total number of pumped energy storage units $\mathrm{j}$ for all units during period $\mathrm{t}$ The total output; $\mathrm{Ni}$ is the total number of thermal power units; $P_{\text {Git }}$ is the output power of the thermal power unit $i$ in the period t. This article does not consider the network loss and network restrictions, and believes that the system is capable of accepting a certain percentage of wind power into the grid in full. System standby constraints.

$\sum_{i=1}^{N_{i}} u_{i t}\left(P_{G \max , i}-P_{G i t}\right)+\sum_{j=1}^{N_{t}}\left(P_{H \max , j}-P_{H j t}\right) \geq k_{d} P_{D t}+k_{w} \sum_{k=1}^{N_{w}} P_{W k t}$

In the formula, $P_{\text {Gmax }}, \mathrm{i}$ is the maximum output power of thermal power unit $\mathrm{i}$; $P_{H \max }, \mathrm{j}$ is the maximum output power of hydropower station $\mathrm{j}$; $\mathrm{kd}$ and $\mathrm{kw}$ are the coincidence fluctuation coefficient and the wind power fluctuation coefficient, respectively, taking $10 \%$ and $15 \%$. The system requires the reserve capacity to meet the random fluctuations of wind power and load.

2.2.3 Electric vehicle cluster energy storage unit model and constraints 
According to the owner's travel and electric vehicle SOC, three control modes are formulated: travel mode, regulation mode, and standby mode.

(1) Travel mode: electric vehicles are charged when the state of charge is lower than $90 \% \mathrm{SOC}$, and participate in grid regulation or wait when the state of charge is higher than $90 \%$ SOC;

(2) Regulation mode: the electric vehicle is charged when the state of charge is lower than the preset SOC, and participates in grid regulation or waiting when the state of charge is higher than the preset SOC, as shown in Figure 2:

(3) Standby mode: The user has no travel demand in the near future, and the electric vehicle fully participates in the regulation of the grid, and the electric vehicle is charged when the state of charge of the electric vehicle is lower than the preset SOC lower than $25 \%$. Stop charging when it is higher than $90 \%$, and between $25 \%$ and $90 \%$, electric vehicles participate in regulation or wait.

Electric vehicles in cities are mainly buses, taxis and private cars. Among them, the number of private cars accounted for most of the total number of electric vehicles. The urban private car mileage and the initial state of charge of electric vehicles are linearly inversely correlated, and logarithmically distributed. Figure 3 shows the initial state of charge of electric vehicles during working days. The probability density, calculated from Figure 3, shows that $92.4 \%$ of users have a state of charge higher than 0.6 , and users higher than 0.8 account for $78.6 \%$. During the network access period 【sjmin,sjmax 】 is the range of SOC that electric vehicles can output, $\mathrm{Sj}, \mathrm{x}$ is the $\mathrm{SOC}$ value required by the car owner to travel. Modeling the energy storage capacity of single electric vehicles, the real-time SOC of electric vehicle batteries and supercapacitors are as follows: 
$\left\{\begin{array}{c}S_{j}(t)=S_{j, s}+\int \frac{P_{j}(t) \eta_{j}(t)}{R_{j}(t)} d t \\ Q_{j}(t)=(1-\delta) Q_{j}(t-1)+P_{j} \Delta t\end{array}\right.$

Where $\sigma_{j}$ is the supercapacitor self-discharge rate; $P_{j}(t)$ is the charge and discharge power at time $\mathrm{t}, Q_{j}(t-1)$ is the remaining capacity of the supercapacitor at time $\mathrm{t}-1$, and $\Delta \mathrm{t}$ is the difference between time $t_{n-1}$ and time $t_{n}$ Time interval. The mathematical model of the relationship between the remaining capacity of the charging and discharging power of the electric vehicle battery equipment and its SOC during the working period.

$\operatorname{SOC}_{j}(t)=\left\{\begin{array}{c}\operatorname{SOC}_{j}(t-1)+\int_{t-1}^{t} \frac{P_{j}(t)}{Q_{E}} d t \\ \operatorname{SOC}_{j}(t-1)+\int_{t-1}^{t} \frac{P_{j}(t) \eta_{j, c}}{\eta_{j, f} Q_{E}} d t\end{array}\right.$

In the formula, $\operatorname{SOC}_{j}(t-1)$ is the SOC of the battery at $\mathrm{t}-1, Q_{E}$ is the capacity of the battery, $\eta_{j, f}$ and $\eta_{j, c}$ are the discharge and charging efficiency of the battery, $P_{j}(\mathrm{t})$ Is the real-time output power of the battery at time t, $P_{j}(\mathrm{t})>0$ means charging, and $P_{j}(\mathrm{t})<0$ means discharging. Taking into account the travel demand of single electric vehicles, the energy storage capacity of single electric vehicles is modeled, as shown in equation (3):

$$
\left\{\begin{array}{c}
R_{j, c}=\left(S O C_{j, z}-S O C_{j, x}\right) R_{j} \\
R_{j, f=}\left(S O C_{j, x}-S O C_{j, y}\right) R_{j}
\end{array}\right.
$$

Where $R_{j, c}$ is the chargeable storage capacity of electric vehicles, $R_{j, f}$ is the dischargeable energy storage capacity of electric vehicles, and $R_{j}$ is the total capacity of electric vehicles. Under constraints:

$$
\left\{\begin{array}{l}
S_{j, \max }=\max \left\{R_{j}, R_{j, c} \text { or } R_{j, f}\right\} \\
S_{j, \min }=\min \left\{R_{j}, R_{j, c} \text { or } R_{j, f}\right\}
\end{array}\right.
$$

The cluster energy storage system of electric vehicles is based on the energy storage capacity model of single electric vehicles. From the number of network access 
$N_{A}$ clusters at time $\mathrm{t}$, the system calculates the overall energy storage capacity of electric vehicles to form a cluster energy storage system. The definition $\operatorname{set}_{A}=\left\{1, \ldots, j-1, j, j+1, \ldots, n_{a}(t)\right\}$ is the $\mathrm{k}$ set of electric vehicles, and the energy storage capacity and SOC of the electric vehicle cluster are as follows:

$$
\begin{aligned}
& \left\{\begin{array}{l}
R_{A, c}^{k}(t)=\sum_{j=1}^{N_{A}^{k}(t)} R_{j, c}^{k}(t) \\
R_{A, f}^{k}(t)=\sum_{j=1}^{N_{A}^{k}(t)} R_{j, f}^{k}(t)
\end{array}\right. \\
& \left\{\begin{array}{l}
S_{A, c}^{k}(t)=\sum_{j=1}^{N_{A}^{k}(t)} \frac{R_{j, c}^{k}(t)}{R_{j}(k)} \\
S_{A, f}^{k}(t)=\sum_{j=1}^{N_{A}^{k}(t)} \frac{R_{j, f}^{k}(t)}{R_{j}(k)}
\end{array}\right.
\end{aligned}
$$

Where $R_{A}$ is the equivalent battery energy storage capacity of the electric vehicle cluster; $S_{A}$ is the equivalent battery SOC value of the cluster. In order to make full use of the energy storage capacity of electric vehicles and reduce the impact of tie-line power fluctuations on the grid, a power adaptive allocation algorithm is proposed Stabilization control strategy, the realization of coordinated control is mainly divided into the following three steps.

Determine the total output power of multiple electric vehicle clusters, that is, the tie line power leveling target value

If $P_{D}(t)>P_{w}(t)$, then:

$$
\begin{aligned}
& P_{c h}(t)=P_{c h}(t-\Delta t)+\left[P_{D}(t)-P_{w}(t)\right] \\
& \text { If } P_{D}(t)=P_{w}(t), \text { then: } \\
& P_{c h}(t)=P_{c h}(t-\Delta t)
\end{aligned}
$$

If $P_{D}(t)<P_{w}(t)$, then:

$$
P_{c h}(t)=P_{c h}(t-\Delta t)+\left[P_{D}(t)-P_{w}(t)\right]
$$

Determine the target change value of the total output of the electric vehicle cluster $\Delta P_{c h}(t)$. According to the different cluster energy storage capabilities of different electric vehicles, use the following procedures (a) (d) to determine the target change value of the cluster output power.

If $0 \leq \Delta P_{c h}(t) \leq \sum_{k=1}^{N_{A}} R_{j, c}^{k}(t)$, clusters with larger energy storage capacity are 
allocated to provide more smoothing tasks.

If $\sum_{k=1}^{N_{A}} R_{j, c}^{k}(t) \leq \Delta P_{c h}(t) \leq 0$, assign clusters with smaller energy storage capacity to provide more smoothing tasks.

$\Delta P_{c h}(t)>\sum_{k=1}^{N_{A}} R_{j, c}^{k}(t)$ The cluster energy storage can only complete part of the smoothing, and the remaining tasks are completed by the back energy storage equipment .

$\Delta P_{c h}(t)<\sum_{k=1}^{N_{A}} R_{j, c}^{k}(t)$ Cluster energy storage can only complete part of the smoothing, and the remaining tasks are completed by the back energy storage equipment, City total load curve under different penetration rates is shown in Figure7.

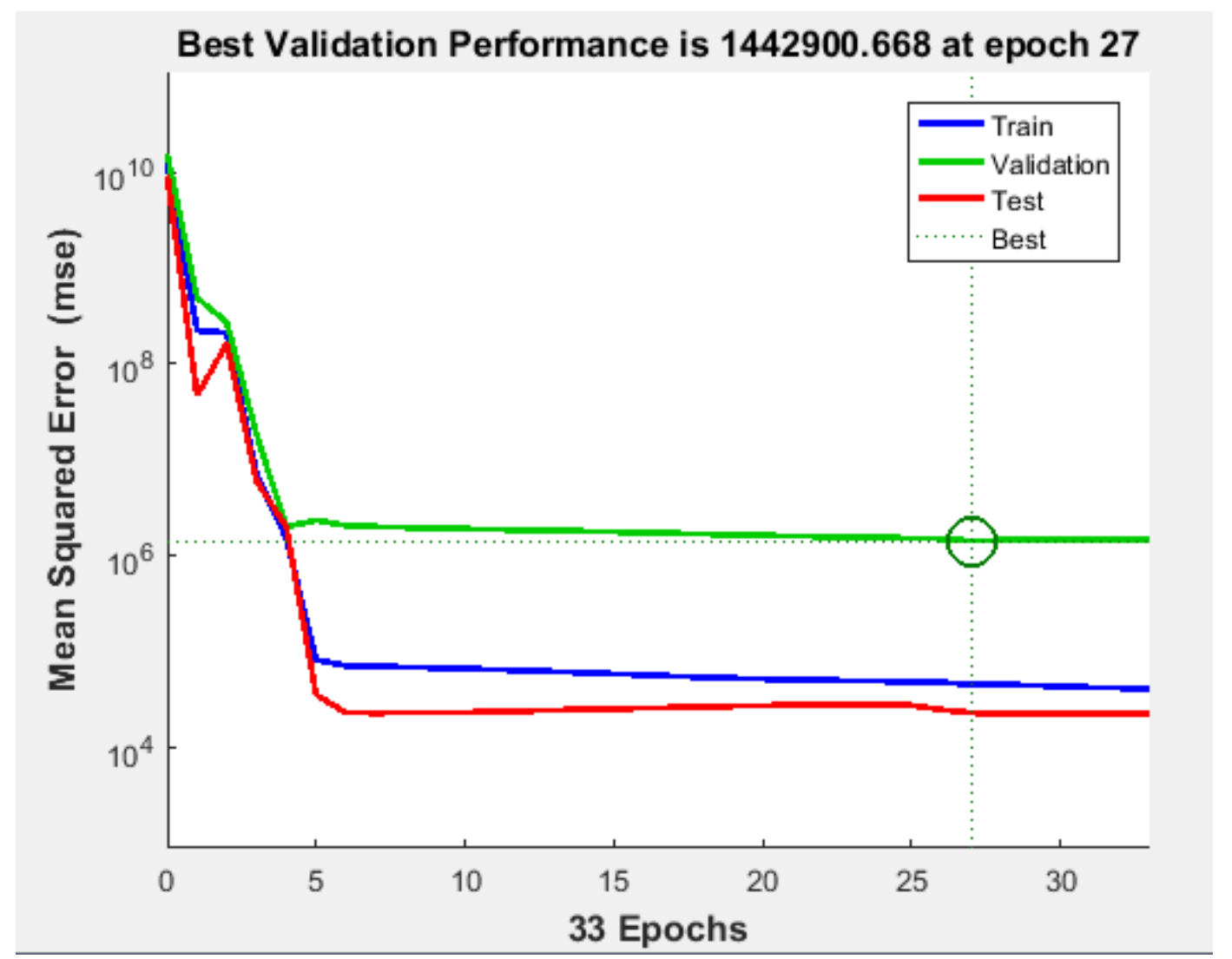

Fig.7. City total load curve under different penetration rates

Make full use of the cluster energy storage capacity of electric vehicles, smooth power fluctuations, consider the upper and lower limits of electric vehicle output power 
and SOC constraints, and determine the output power of electric vehicles $P_{j, f}^{k}$.

$0 \leq \Delta P_{c h}(t) \leq \sum_{k=1}^{N_{A}} R_{j, c}^{k}(t)$ Considering the high cost of discharging electric vehicles, electric vehicles take less discharge tasks during the control process. At this time, the power adaptive allocation algorithm is used to preferentially call the backup energy storage to supplement the discharge capacity. Electric vehicles with higher SOC provide more power distribution as follows:

$$
P_{j, f}^{k}=P_{j}^{k}(t)+\frac{S_{j, z}^{k}(t)-S_{j, x}^{k}(t)}{\sum_{j=1}^{N_{A}^{K}}\left(S_{j, z}^{k}(t)-S_{j, x}^{k}(t)\right)} \times\left(\nabla P_{c h}^{k}(t)-\nabla P_{c}(t)\right)
$$

If $\Delta P_{c}(t)>0$, electric vehicles with lower SOC provide more power allocation as follows:

$$
\begin{aligned}
& P_{j, c}^{k}=P_{j, k}^{t}+\frac{s_{j, y}^{k}(t)-s_{j, x}^{k}(t)}{\sum_{j=1}^{N_{A}^{k}}\left(S_{j, z}^{k}(t)-S_{j, x}^{k}(t)\right)} \times \nabla P_{c h}^{k}(t) \\
& P_{j}^{k}(t)>P_{j, \max }^{k}(t), \text { Amend } P_{j, y}^{k}(t)=P_{j, \max }^{k}(t) \\
& P_{j}^{k}(t)<P_{j, \max }^{k}(t), \text { Amend } P_{j, y}^{k}(t)=P_{j, \min }^{k}(t)
\end{aligned}
$$

Therefore, the total output power of electric vehicle cluster energy storage is: $P=$ $\sum_{k=1}^{m} \sum_{j=1}^{N_{A}^{k}} P_{j}^{k}(t)$, cluster energy storage system The energy management coordination control strategy is mainly divided into three modes to allocate energy,

Large-scale disorderly electric vehicles entering the grid not only did not play a good role in shaving the peak and filling valleys of the original load, but aggravated the fluctuation of the load to a certain extent. After the electric vehicles entered the grid, the overall electricity load of the urban distribution network was increased. It poses higher challenges to the reliability, economy, and safety of the distribution network.

Therefore, in this article, the power fluctuation of the tie line is the research object, aiming at the fluctuation of wind power output, and reducing the load pressure of the distribution network, using electric vehicle groups in various regions to stabilize the power fluctuation of the tie line to provide energy for the city. The article mainly focuses on the functional energy. Scheduling. The use of stabilization control strategies and the use of electric vehicle cluster energy storage to effectively smooth the power 
fluctuations of the tie-line, effectively reduce the power fluctuations of wind power access, smooth wind power grid connection to improve wind power consumption, and reduce the impact of large-scale electric vehicles randomly accessing the grid on electric vehicles.Accurate modeling of single electric vehicles, combined with network access characteristics, and taking into account the travel needs of vehicle owners, assessing the cluster energy storage capacity of electric vehicles, equipped with adaptive power allocation algorithms, and enhancing the ability to adjust the output power of electric vehicles. The use of electric vehicle clusters to build a cluster energy storage system, so that the fixed energy storage equipment system is only used as a backup resource, which effectively reduces the requirements for the capacity of the energy storage system for wind power access to the grid. City total load curve under different scenarios is shown in Figure 8.

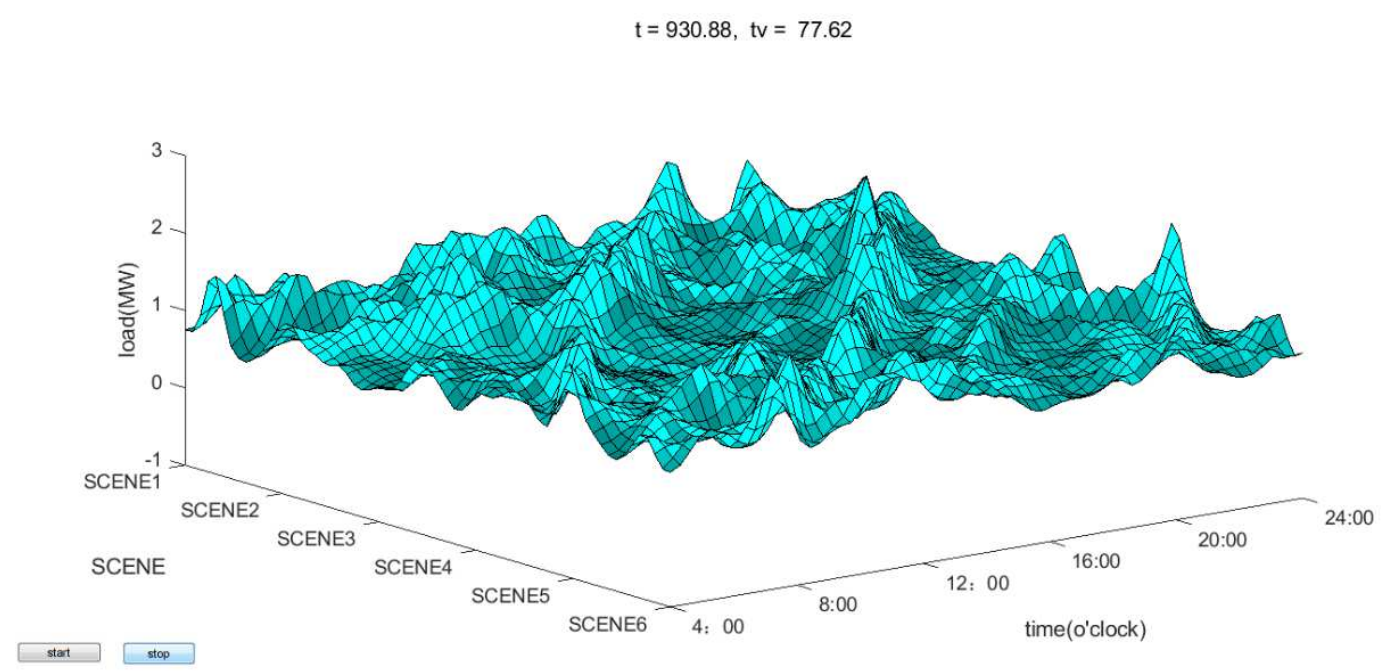

Fig.8. City total load curve under different scenarios

\subsection{Photovoltaic water pump}

The basic structure of the photovoltaic water pump system. The photovoltaic water pump system is a system that integrates "light, machine, electricity, materials" and other multidisciplinary high-tech systems. It is not only applied to traditional technologies such as photovoltaic materials, solar energy harvesting, power electronic conversion, and motor drive control. The results will be applied to super management systems such as the Internet of Things and cloud platforms in the near future. Photovoltaic water pump systems have high practical value in domestic water, seawater desalination, 
desert management, agricultural irrigation, grassland animal husbandry, scenic fountains, sewage treatment, etc. The core technology of photovoltaic water pump system lies in two parts: AC and DC power conversion and water pump motor drive control.

According to the working characteristics of the photovoltaic water pump, by adjusting the rotation speed of the load of the water pump, the working point of the photovoltaic array can be controlled, so that the system can output the maximum power stably. For an asynchronous motor, the rotation speed can be adjusted by changing the voltage and frequency. From the above-mentioned T-type equivalent circuit of the asynchronous motor, the voltage of each phase of the stator of the three-phase asynchronous motor can be obtained. As long as $\mathrm{U} 1$ and $\mathrm{fl}$ are controlled, the magnetic flux can be controlled. Three-phase asynchronous motor variable voltage frequency conversion speed regulation has the following characteristics: (1) Speed regulation from the base frequency downwards, keeping the magnetic flux constant, which is a constant torque speed regulation method; from the fundamental frequency upwards, the magnetic flux and The frequency decreases in inverse proportion, which is approximately a constant power speed regulation mode; (2) The motor frequency f can be continuously adjusted. The three-phase asynchronous motor is a non-linear, multivariable, strong coupling, multi-parameter system, which is difficult to accurately control by simple external control signals.

When the temperature is constant, the maximum output power of the photovoltaic array increases with the increase of the light intensity. Since the power of the water pump in the photovoltaic water pump system is approximately proportional to the cube of the speed, and the speed is proportional to the frequency, adjusting the output frequency of the inverter power supply is equivalent to adjusting the power of the load motor. The controller adopts an optimized sliding mode control strategy, a fixed duty cycle control in the steady state of MPPT, and an optimized sliding mode control in the tracking state. This control strategy can effectively reduce power chattering. The power chattering curve of the optimized sliding mode control method is smoother than that in the literature, and the chattering is smaller. Through the simulation data, we can know 
that the optimized sliding mode control MPPT technology can quickly track the maximum power point. When the external light intensity changes suddenly, it not only has a faster dynamic response speed, but also has a smaller chattering.

After the bus voltage setting value is compared with the DC voltage measured by the photovoltaic array feedback, the deviation value is passed through the PI regulator to obtain the frequency control signal. The frequency signal is converted into the motor stator reference phase voltage through the voltage-frequency ratio curve, and then pulses are generated by the SVPWM generator Signal to control the duty cycle D of the power switch tube of the IPM module to adjust the output voltage and frequency of the inverter. The algorithm mainly includes the following steps:

Step1. Determine the sector where the composite vector is located;

Step2. Calculate the action time of two adjacent vectors in real time;

Step3. Determine the conduction time of each bridge arm;

Step4. Obtain the PWM duty cycle D of each phase;

Step5. Update the corresponding register value.

The commonly used water pumps in photovoltaic water pumping systems mainly include positive displacement pumps and centrifugal pumps. The working principle of positive displacement pumps is to pump water through periodic changes in the internal volume of the pump; centrifugal pumps use the centrifugal force generated by the highspeed rotation of the impeller to form a vacuum at the impeller inlet, Press the water into the pipeline to complete the pumping under the action of atmospheric pressure. Simply put, positive displacement pumps work by volume conversion, and centrifugal pumps work by centrifugal force. The user determines the type and power level of the pump according to the actual use conditions, mainly according to the different requirements of flow, head, and efficiency. Using the methods we have researched can greatly improve the utilization of wind and solar energy, reduce dependence on fossil energy, and reduce carbon emissions. The later research plan will combine sensor technology to automatically detect soil moisture, nutrient elements, and light conditions. Watering plants and supplementing light is like installing a brain for plants that can manage their own growth environment. Professor Liu Zuming's team also realized the 
completion of the photovoltaic water pumping system with the highest lift in the world. The project of emergency water pumping for drought relief on the Jinsha River in Jiangyi Township and the consolidation and improvement of rural drinking water safety project has an annual water supply of 410,800 m3, covering Jiangyi Township, Jiangyi Township. The 6 village committees and 41 natural villages of Painter, Banqing, Gongcha, Horizontal Stone and Nuolakun solved the problem of safe drinking water for 11,590 people and 3,140 large livestock in Jiangyi Township. The picture below shows the details of our photovoltaic water extraction

When the temperature is constant, the maximum output power of the photovoltaic array increases with the increase of the light intensity. Since the power of the water pump in the photovoltaic water pump system is approximately proportional to the cube of the speed, and the speed is proportional to the frequency, adjusting the output frequency of the inverter power supply is equivalent to adjusting the power of the load motor. The controller adopts an optimized sliding mode control strategy, a fixed duty cycle control in the steady state of MPPT, and an optimized sliding mode control in the tracking state. This control strategy can effectively reduce power chattering. The power chattering curve of the optimized sliding mode control method is smoother than that in the literature, and the chattering is smaller. Through the simulation data, we can know that the optimized sliding mode control MPPT technology can quickly track the maximum power point. When the external light intensity changes suddenly, it not only has a faster dynamic response speed, but also has a smaller chattering.

After the bus voltage setting value is compared with the DC voltage measured by the photovoltaic array feedback, the deviation value is passed through the PI regulator to obtain the frequency control signal. The frequency signal is converted into the motor stator reference phase voltage through the voltage-frequency ratio curve, and then pulses are generated by the SVPWM generator Signal to control the duty cycle D of the power switch tube of the IPM module to adjust the output voltage and frequency of the inverter. The algorithm mainly includes the following steps:

Step1. Determine the sector where the composite vector is located;

Step2. Calculate the action time of two adjacent vectors in real time; 
Step3. Determine the conduction time of each bridge arm;

Step4. Obtain the PWM duty cycle D of each phase;

Step5. Update the corresponding register value.

The commonly used water pumps in photovoltaic water pumping systems mainly include positive displacement pumps and centrifugal pumps. The working principle of positive displacement pumps is to pump water through periodic changes in the internal volume of the pump; centrifugal pumps use the centrifugal force generated by the highspeed rotation of the impeller to form a vacuum at the impeller inlet, Press the water into the pipeline to complete the pumping under the action of atmospheric pressure. Simply put, positive displacement pumps work by volume conversion, and centrifugal pumps work by centrifugal force. The user determines the type and power level of the pump according to the actual use conditions, mainly according to the different requirements of flow, head, and efficiency. Using the methods we have researched can greatly improve the utilization of wind and solar energy, reduce dependence on fossil energy, and reduce carbon emissions. The later research plan will combine sensor technology to automatically detect soil moisture, nutrient elements, and light conditions. Watering plants and supplementing light is like installing a brain for plants that can manage their own growth environment. Professor Liu Zuming's team also realized the completion of the photovoltaic water pumping system with the highest lift in the world. The project of emergency water pumping for drought relief on the Jinsha River in Jiangyi Township and the consolidation and improvement of rural drinking water safety project has an annual water supply of 410,800 m3, covering Jiangyi Township, Jiangyi Township. The 6 village committees and 41 natural villages of Painter, Banqing, Gongcha, Horizontal Stone and Nuolakun solved the problem of safe drinking water for 11,590 people and 3,140 large livestock in Jiangyi Township. The picture below shows the details of our photovoltaic water extraction is shown in Figure 9. 


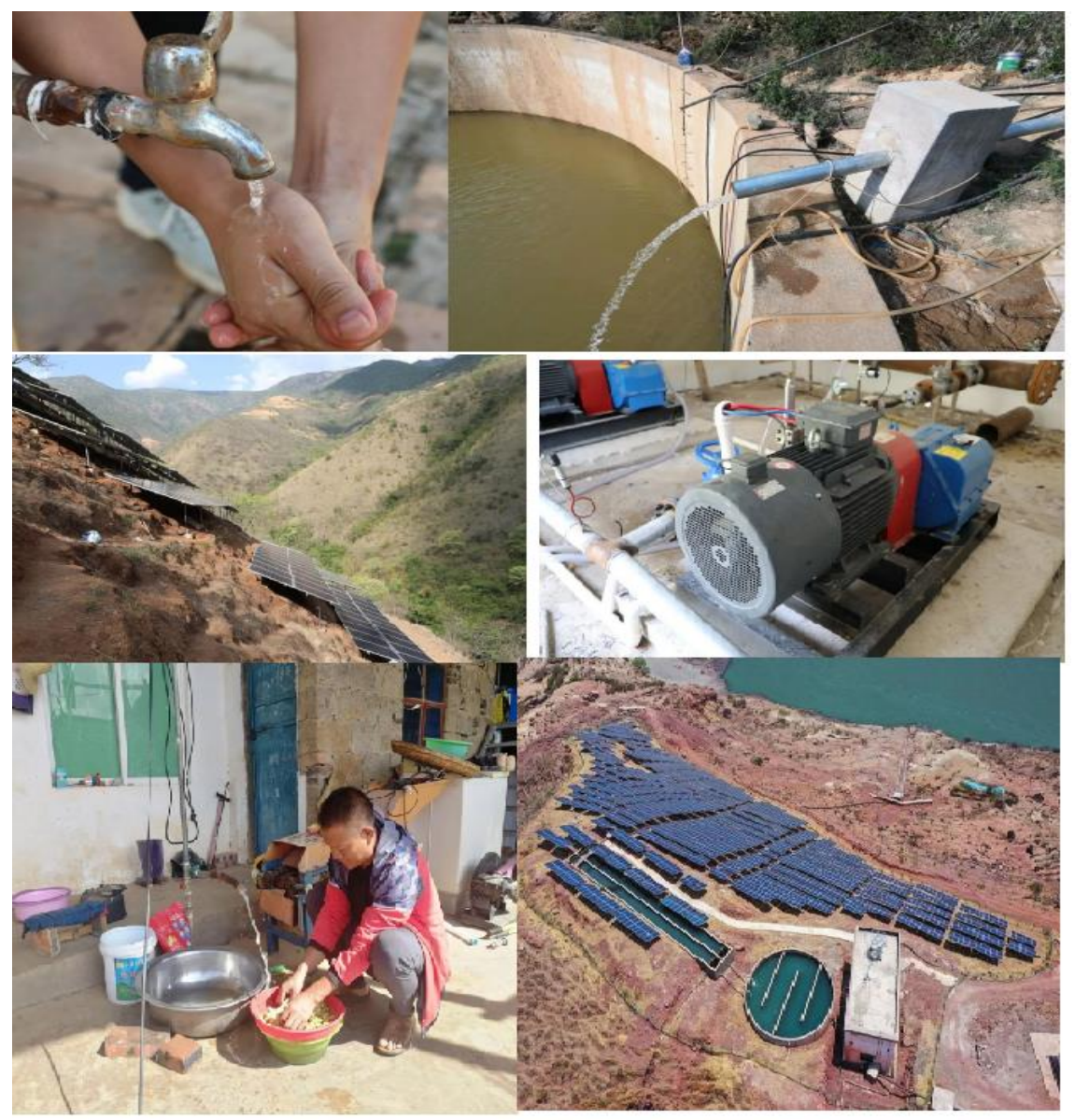

Fig. 9. photovoltaic water pump case

\subsection{The relationship between energy transition and carbon dioxide}

\subsubsection{The impact of the system on carbon dioxide emissions}

The use of fossil energy such as coal and petroleum provides basic impetus for economic and social development, but also produces pollutants such as sulfides, nitrides, inhalable particulate matter and $\mathrm{CO} 2$, causing environmental pollution and global climate change, thus promoting energy Transformation has always been a hot and frontier issue in the international community. Since 2006, my country has become the world's largest energy consumption and CO2 emitter. In 2019, coal consumption accounted for $51.7 \%$ of the world's total and $\mathrm{CO} 2$ emissions accounted for $28.8 \%$ of the world's total. The coal-based energy structure is facing domestic requirements to improve the environment, International pressure to reduce $\mathrm{CO} 2$ emissions. In addition, my country has a high degree of dependence on foreign oil and natural gas, and energy security is facing potential threats. Therefore, promoting my country's energy transition 
and optimizing the energy structure are issues that urgently need to be resolved in the sustainable and coordinated development of energy, economy, and environment. The internal mechanism of the energy revolution promoting the rise of high quality in Central China is shown in Figure 10.

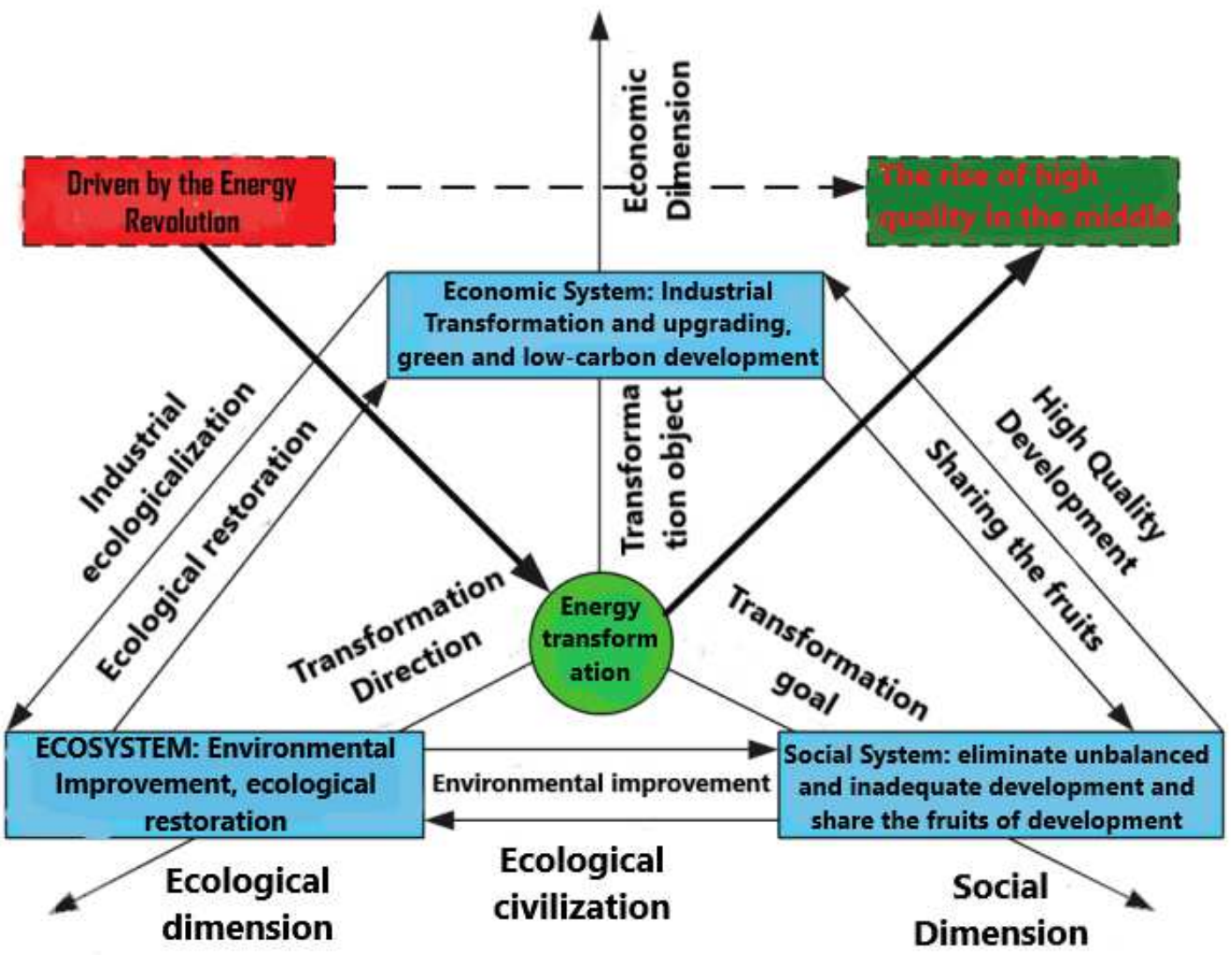

Fig.10. The internal mechanism of the energy revolution promoting the rise of high quality in Central China

2.4.2 Scenario design of energy revolution boosting energy structure transformation

The core of the energy revolution is the development of clean and lowcarbon energy, which is manifested in changes in energy structure in terms of energy consumption. The energy consumption structure of the five central provinces is dominated by coal, and coal consumption accounts for a relatively high proportion of total energy. In recent years, driven by technological innovation, the proportion of direct coal consumption has shown a downward trend, and the clean conversion rate of coal has been greatly improved; various provinces have vigorously developed renewable 
energy such as wind energy, solar energy, hydropower, and biomass energy. Form a multi-energy system such as wind-solar complementation, water-light complementation, etc. In regional energy cooperation, "West-East Gas Transmission", "West-East Electricity Transmission", "North Coal-South Transportation", UHV smart grids, etc., have provided energy structure transformation in the central region. Facilitating conditions; accordingly, a scenario where the energy revolution promotes the transformation of the energy structure in the central region has been constructed (see Table 2).

Table2Energy structure scenarios of the five central provinces

Table 2 Energy structure scenarios of the five central provinces

\begin{tabular}{|c|c|c|c|c|c|c|c|c|c|}
\hline \multirow[t]{2}{*}{ province } & \multirow[t]{2}{*}{ Time/year } & \multicolumn{2}{|c|}{$\begin{array}{l}\text { Baseline } \\
\text { scenario/\% }\end{array}$} & \multicolumn{2}{|c|}{ development } & \multicolumn{4}{|c|}{ Energy revolution scenario/\% } \\
\hline & & coal & oil & $\begin{array}{l}\text { natural } \\
\text { gas }\end{array}$ & $\begin{array}{l}\text { Renewable } \\
\text { Energy }\end{array}$ & coal & oil & $\begin{array}{l}\text { natural } \\
\text { gas }\end{array}$ & $\begin{array}{l}\text { Renewable } \\
\text { Energy }\end{array}$ \\
\hline \multirow{4}{*}{$\begin{array}{l}\text { Henan } \\
\text { Province }\end{array}$} & 2020 & 68.3 & 15.9 & 6.8 & 9.0 & 68 & 15.9 & 6.8 & 9.3 \\
\hline & 2025 & 57.7 & 19.5 & 8.5 & 14.2 & 57.4 & 19.5 & 8.5 & 14.5 \\
\hline & 2030 & 48.7 & 20.7 & 10.6 & 20.0 & 48.4 & 20.7 & 10.6 & 20.3 \\
\hline & 2035 & 41.4 & 21.3 & 13.4 & 23.9 & 40.9 & 21.3 & 13.4 & 24.4 \\
\hline \multirow{4}{*}{$\begin{array}{l}\text { Anhui } \\
\text { Province }\end{array}$} & 2020 & 70.5 & 18.0 & 5.2 & 6.3 & 70.0 & 18.0 & 5.2 & 6.8 \\
\hline & 2025 & 66.4 & 18.2 & 8.1 & 7.3 & 63.8 & 18.2 & 8.1 & 9.9 \\
\hline & 2030 & 61.5 & 18.4 & 9.3 & 11.0 & 58.0 & 18.4 & 9.3 & 14.3 \\
\hline & 2035 & 56.0 & 18.6 & 10.1 & 15.3 & 53.3 & 18.6 & 10.1 & 18.0 \\
\hline \multirow{4}{*}{$\begin{array}{l}\text { Hubei } \\
\text { Province }\end{array}$} & 2020 & 47.3 & 21.7 & 5.4 & 25.6 & 47.0 & 21.7 & 5.4 & 25.9 \\
\hline & 2025 & 43.4 & 21.9 & 8.8 & 25.9 & 43.1 & 21.9 & 8.8 & 26.2 \\
\hline & 2030 & 37.4 & 22.2 & 14.2 & 26.2 & 37.1 & 22.2 & 14.2 & 26.5 \\
\hline & 2035 & 32.5 & 22.5 & 18.5 & 26.5 & 32.5 & 22.5 & 18.5 & 26.8 \\
\hline \multirow{4}{*}{$\begin{array}{l}\text { Hunan } \\
\text { Province }\end{array}$} & 2020 & 63.7 & 16.4 & 3.0 & 16.9 & 63.4 & 16.4 & 3.0 & 17.2 \\
\hline & 2025 & 60.3 & 16.6 & 4.8 & 18.3 & 60.0 & 16.6 & 4.8 & 18.6 \\
\hline & 2030 & 55.6 & 16.9 & 7.8 & 19.7 & 55.3 & 16.9 & 7.8 & 20.0 \\
\hline & 2035 & 51.8 & 17.1 & 10.1 & 21.0 & 51.5 & 17.1 & 10.1 & 21.3 \\
\hline \multirow{4}{*}{$\begin{array}{l}\text { Jiangxi } \\
\text { Province }\end{array}$} & 2020 & 68.8 & 18.2 & 4.1 & 8.8 & 68.5 & 18.2 & 4.1 & 9.1 \\
\hline & 2025 & 63.1 & 18.5 & 6.7 & 11.7 & 62.8 & 18.5 & 6.7 & 12.0 \\
\hline & 2030 & 53.4 & 18.7 & 10.8 & 17.1 & 53.1 & 18.7 & 10.8 & 17.4 \\
\hline & 2035 & 44.6 & 19.0 & 14.0 & 22.4 & 44.3 & 19.0 & 14.0 & 22.7 \\
\hline
\end{tabular}


2.4.3 Path choice for the energy revolution to boost the rise of the central region

(1) Energy demand of different paths in the rise of the central region

Integrate various macro overall factors in the process of the rise of the central region, such as economic growth, industrial development, new urbanization, energy efficiency, per capita income, and living energy, into different options for development paths, and obtain different options through policy guidance and implementation. Development path and corresponding effects. According to different scenarios, the energy demand of the five central provinces under different paths from 2020 to 2035 can be obtained.

Take the scenario of high economic growth as an example (see Figure 2). By 2035, the rising energy demand in the central region will show an increasing trend without an inflection point, and the energy demand will be about $8.45 \times 10^{8} \sim 1.0 \times 10^{9}$ tce. For example, if the energy revolution is adopted to promote industrial structure optimization and energy efficiency improvement (SE11 path), the energy demand in 2035 will be approximately $9.12 \times 10^{8}$ tce; if the energy revolution cannot promote industrial structure optimization and energy efficiency improvement, the urbanization process will accelerate (SE14 path) ), the energy demand is about $1.0 \times 10^{9}$ tce; if you choose one of the two to promote the upgrading of industrial structure and improve energy efficiency, it is the SE12 or SE13 route, but the energy demand of the SE13 route is $4.55 \times 10^{7}$ lower than that of the SE12 route tce, this shows that the improvement effect of energy technology efficiency is higher than that of industrial structure optimization. Future carbon dioxide emissions under different scenarios Figure11 Energy demand under high economic growth scenarios 


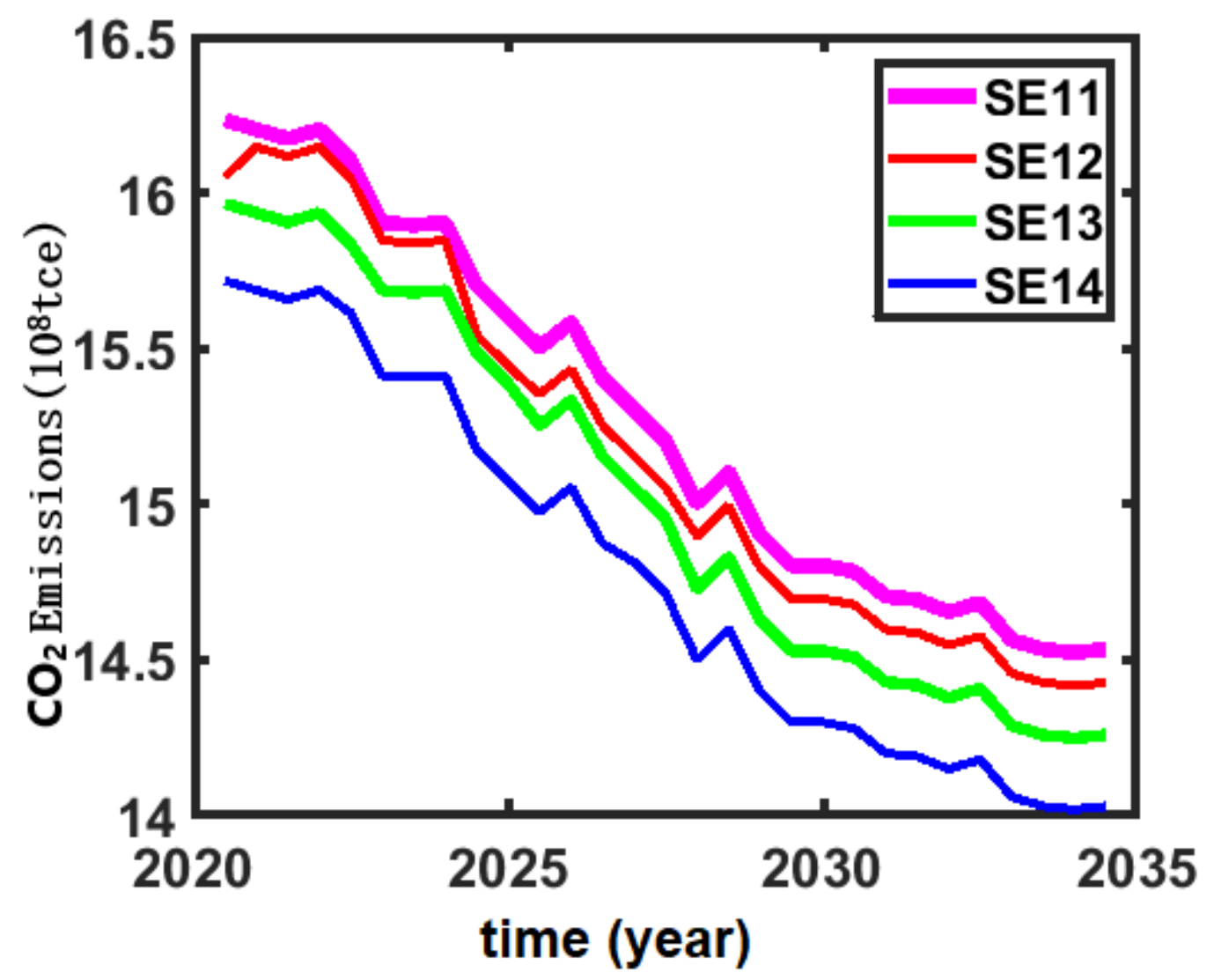

Fig.11. Future carbon dioxide emissions under different scenarios Figure 2 Energy demand under high economic growth scenarios

In the context of benchmark economic growth, by 2035, the energy demand of the five central provinces will be approximately $9.01 \times 10^{8} \sim 9.74 \times 10^{8}$ tce. In the case of industrial structure upgrade and energy efficiency improvement (SE24 path), the energy demand in the central region is about $9.01 \times 108$ tce; if the energy revolution cannot promote the upgrading of the industrial structure and improve energy efficiency, the energy demand is about $9.74 \times 10^{8}$ tce; other situations are somewhere in between In the energy revolution, if the industrial structure is adjusted and energy efficiency is synchronized (SE22 path), the energy demand is about $9.11 \times 10^{8}$ tce; if only the industrial structure upgrade is promoted without improving energy efficiency (SE23 path), the energy demand is about $9.60 \times 10^{8}$ tce; in the context of accelerating urbanization, the industrial structure and energy efficiency remain unchanged (SE25 path), and the energy demand is about $9.58 \times 10^{8}$ tce. 
Under the background of low economic growth, by 2035, energy demand is divided into four situations: the baseline scenario (SE31 path), the energy demand is about $9.11 \times 10^{8}$ tce; the baseline scenario and the energy revolution improves energy efficiency (SE32 path), energy The demand is about $8.57 \times 10^{8}$ tce; the additional energy revolution of the SE32 path accelerates the optimization and upgrading of the industrial structure (the SE33 path), and the energy demand is about $8.99 \times 10^{8}$ tce; the additional energy revolution of the SE33 path not only promotes the optimization of the industrial structure but also improves energy efficiency. The demand is about $8.45 \times 10^{8}$ tce.

\section{Conclusions}

It can be seen that the combination of the energy revolution strategy and the central region's rise strategy will help promote the high-quality development of the central region. The energy revolution promotes the transformation and upgrading of the industrial structure in the central region by transforming the energy structure and improving energy efficiency, and plays a positive role in the development of new urbanization in the region and the improvement of residents' living standards. Under the conditions of comprehensive consideration of economic growth, industrial development, urbanization, and existing energy-saving policies, the energy demand in the central region will continue to rise along with the advancement of industrialization and urbanization. There will be no turning point before 2035, but energy efficiency will increase. The optimization of industrial structure will help reduce energy demand; the energy revolution scenario with the goal of building a diversified and complementary energy system will help the central region to reach the peak time of carbon as soon as possible, and promote the coordinated development of the economy, society, and environment in the central region. In response to the background of the rise of the central region, the following development suggestions are put forward for the regional energy revolution.

(1) Focus on the development of energy-saving and clean technologies to improve energy efficiency and clean and low-carbon energy supply

Energy consumption in the central region is dominated by coal, and the dominant 
position of coal in primary energy is difficult to change in the short term. Since thermal power is the main method of coal conversion in my country, the development of thermal power technology innovation is an important aspect of the development of related clean technologies. The clean use of coal is the focus of the future energy revolution, and energy saving and efficiency improvement is the first of my country's energy strategy; on the one hand, it will increase the upgrading of thermal power units and the elimination of small-scale units in the central region, and improve the energy input and output efficiency of various industries in the region. Carry out regulation and continuously improve the efficiency of energy utilization; on the other hand, plan and implement the development of renewable energy such as hydropower, wind power, photovoltaic, and biomass energy.

(2) Intensify the adjustment of industrial structure and transform to green, lowcarbon and ecological, The energy revolution promotes the rise of the central region, which is not only a problem of the energy industry structure itself, but also a problem of advanced and ecological development of the entire industrial structure in the region. Under the new concept of ecological priority and green development, the central region should change the traditional extensive development method that relies on resources, focus on industrial transformation and upgrading, promote industrial restructuring according to local conditions, and develop towards low-carbon ecological development. It is recommended to incorporate the construction of ecological civilization into the process of industrial transformation and upgrading, develop new industrialization to adjust and optimize the industrial structure; change the industrial model of "resourcesproducts-waste discharge" of industrial civilization, and gradually shift to the development mode of ecological civilization and harmonious coexistence of man and nature; adopt advanced Technological transformation and upgrading of traditional industries, implementation of traditional technological transformation and upgrading, and the use of information technology and new energy technology to promote the ecological development of industries.

(3) Promote the reform of the energy system and build a multi-competitive market 
system, The development and growth of new energy is inseparable from market breakthroughs. my country's current "coal-electricity joint operation" and "coalelectricity integration" operating system has actually formed the monopoly of coal power on the grid, and new energy sources such as wind and photovoltaics are basically available in price. Conditions to compete with traditional thermal power. It is recommended to make use of the decentralized characteristics of new energy, combine with the construction of "beautiful villages" and "safe communities", adopt a development path from rural to urban, from life to production, design a competitive market, and better play the decisive role of the market in resource allocation . In view of the regional differences in the distribution of wind and solar energy, it is recommended that the provinces in the central region establish a consultation mechanism, break administrative divisions, and fully strengthen regional cooperation in the use of new energy; promote energy system reform, break institutional constraints and break regional gaps, and advocate renewable energy Prioritize the development and utilization of the environment, and better implement the concept of ecological priority development.

\section{References}

[1]Almeida, S.M., Silva, A., Sarmento, S., 2014. Effects of exposure to particles and ozone on hospital admissions for cardiorespiratory diseases in setúbal, Portugal. J. Toxicol. Environ. Health, Part A 77 (14-16), 837-848.

[2]B.B. Wei, G. Mei, H.F. Liang, Z.B. Qi, D.F. Zhang, H. Shen, Z.C. Wang, Porous $\mathrm{CrN}$ thin films by selectively etching $\mathrm{CrCuN}$ for symmetric supercapacitors, J. Power Sources 385 (2018) 39-44.

[3]B. Subramanian, R. Ananthakumar, M. Jayachandran, Effect of substrate temperature on the properties of reactively sputtered TiN/NbN multilayers, Cryst.Res. Technol. 46 (2011) 1273-1282.

[4]Chatzidiakou, L., Krause, A., Popoola, O.A., Antonio, A.D., Kellaway, M., Han, Y.,Squires, F.A., Wang, T., Zhang, H., Wang, Q., et al., 2019. Characterising low-cost sensors in highly portable platforms to quantify personal exposure in diverse environments. Atmospheric measurement techniques 12 (8), 4643-4657. 
[5]Devarakonda, S., Sevusu, P., Liu, H., Liu, R., Iftode, L., Nath, B., 2013. Realtime air quality monitoring through mobile sensing in metropolitan areas. In: Proceedings of the 2nd ACM SIGKDD International Workshop on Urban Computing, pp. $1-8$.

[6]D. Aaron, Q. Liu, Z. Tang, G. Grim, A. Papandrew, A. Turhan, T. Zawodzinsk i, M. Mench Dramatic performance gains in vanadium redox flow batteries through modified cell architecture J. Power Sources, $206(2012)$, pp. 450453, 10.1016/j.jpowsour.2011.12.026

[7]G.L. Soloveichik Flow batteries: current status and trends Chem. Rev., 115 (2015), pp. 11533-11558, 10.1021/cr500720t CrossRefView Record in ScopusGoogle Scholar

[8]H.-G. Schweiger, O. Obeidi, O. Komesker, A. Raschke, M. Schiemann, C. Zehner,M. Gehnen, M. Keller, P. Birke, Sensors 10 (6) (2010) 5604-5625.

[9]H.L. Cui, G.L. Zhu, X.Y. Liu, F.X. Liu, Y. Xie, C.Y. Yan, T.Q. Lin, H. Gu, F.Q. Huang,Niobium nitride Nb4N5 as a new high-performance electrode material for supercapacitors, Adv. Sci. 2 (2015), 1500126.

[10]J. Chang, M.H. Jin, F. Yao, T.H. Kim, V.T. Le, H.Y. Yue, F. Gunes, B. Li, A. Ghosh, S.S. Xie, Y.H. Lee, Asymmetric supercapacitors based on graphene/MnO2 nanospheres and graphene/MoO3 nanosheets with high energy density, Adv.Funct. Mater. 23 (2013) 5074-5083.

[11]J. Noack, N. Roznyatovskaya, T. Herr, P. Fischer The chemistry of redoxflow batteries angew chem int ed engl, 54(2015), pp.97769809, 10.1002/anie.201410823

CrossRefView Record in ScopusGoogle Scholar

[12]Kaliszewski, M., Włodarski, M., Mły'nczak, J., Kopczy’nski, K., 2020. Comparison of lowcost particulate matter sensors for indoor air monitoring during covid-19 lockdown.Sensors 20 (24), 7290.

[13]Luxen, D., Vetter, C., 2011. Real-time routing with openstreetmap data. In: Proceedings of the 19th ACM SIGSPATIAL International Conference on Advances in Geographic Information Systems, GIS '11. ACM, New York, NY, USA, pp. 513- 
516.Mariano, P., Almeida, S.M., Santana, P., 2020. Pollution prediction model using

[14]M. Ranjbar, S.M. Mahdavi, A. Iraji zad, Pulsed laser deposition of W-V-O composite films: preparation, characterization and gasochromic studies, Sol. Energy Mater.Sol. Cells 92 (2008) 878-883.

[15]M. Watanabe, K. Sanui, N. Ogata, T. Kobayashi, Z. Ohtaki, Ionic conductivity and mobility in network polymers from poly (propylene oxide) containing lithium perchlorate, J. Appl. Phys. 57 (1985) 123-128.

[16]Mead, M., Popoola, O., Stewart, G., Landshoff, P., Calleja, M., Hayes, M., Baldovi, J.,McLeod, M., Hodgson, T., Dicks, J., et al., 2013. The use of electrochemical sensors for monitoring urban air quality in low-cost, high-density networks. Atmos. Environ.70, 186-203.

[17] P.K. Singh, B. Bhattacharya, R.M. Mehra, H.-W. Rhee, Plasticizer doped ionic liquid incorporated solid polymer electrolytes for photovoltaic application, Curr. Appl.Phys. 11 (2011) 616-619.

[18]P.H. Yang, D.L. Chao, C.R. Zhu, X.H. Xia, Y.Q. Zhang, X.L. Wang, P. Sun, B.K. Tay,Z.X. Shen, W.J. Mai, H.J. Fan, Ultrafast-charging supercapacitors based on cornlike titanium nitride nanostructures, Adv. Sci. 3 (2015), 1500299.

[19]P. Alotto, M. Guarnieri, F. Moro Redox-Flow Batteries for the storage of renewable energy: a review Ren. Sust. Energ. Rev., 29 (2014), pp. 325335, 10.1016/j.rser.2013.08.001 ArticleDownload PDFView Record in ScopusGoogle Scholar

[20]Ripoll, A., Viana, M., Padrosa, M., Querol, X., Minutolo, A., Hou, K.M., Barcelo-Ordinas, J.M., García-Vidal, J., 2019. Testing the performance of sensors for ozone pollution monitoring in a citizen science approach. Sci. Total Environ. 651,11661179.Salimifard, P., Rim,

[21]W. Zhai, H. Zhu, L. Wang, X. Liu, H. Yang, Study of PVDF-HFP/PMMA blended micro-porous gel polymer electrolyte incorporating ionic liquid [BMIM]BF4 for Lithium ion batteries, Electrochim. Acta 133 (2014) 623-630, https://doi.org/ 10.1016/j.electacta.2014.04.076.

[22]W.F. Chen, J.T. Muckerman, E. Fujita, Recent developments in transition 
metal carbides and nitrides as hydrogen evolution electrocatalysts, Chem. Commun. 49(2013) 8896-8909.,

[23]X. Li, H. Peng, M.-S. Wang, X. Zhao, P.-X. Huang, W. Yang, J. Xu, Z.-Q. Wang, M.-Z. Qu, Z.-L. Yu, Enhanced electrochemical performance of Zr-modified layered LiNi1/3Co1/3Mn1/3O2 cathode material for lithium-ion batteries, ChemElectroChem 3 (2016) 130-137.

[24]X. Ke, J.M. Prahl, J.I.D. Alexander, J.S. Wainright, T.A. Zawodzinski, R.F. S avinelli Rechargeable redox flow batteries: flow fields, stacks and design considerations

Chem. Soc. Rev., 47 (2018), pp. 8721-8743, 10.1039/C8CS00072G CrossRefView Record in ScopusGoogle Scholar

[25]Z. Wang, Y. Tang, B. Li, Bicontinuous and cellular structure design of PVDF membranes by using binary solvents for the membrane distillation process, RSC Adv. 8 (2018) 25159-25167.

Acknowledgements

This research was supported by National Natural Science Foundation of China, 51367019

Author contributions

Weiyan $\mathrm{Xu}^{*}$ : Supervision, Funding acquisition, Hardware, Software,Conceptualization, Investigation, Resources, Writing - original draft, Writing - review \& editing.

Zumin Liu: Funding acquisition, Hardware, Conceptualization, Investigation, Resources,Writing - original draft, Writing - review \& editing

.Jielei Tu: Conceptualization, Investigation, Resources,

Competing interests

The authors declare no competing interests.

The image use license means that Party A authorizes Party B to use the images fig1 provided by Party A. 